\title{
Local time penalizations with various clocks for one-dimensional diffusions
}

\author{
By Christophe Profeta, Kouji Yano and Yuko Yano
}

(Received Aug. 30, 2016)

(Revised Aug. 13, 2017)

\begin{abstract}
We study some limit theorems for the law of a generalized one-dimensional diffusion weighted and normalized by a non-negative function of the local time evaluated at a parametrized family of random times (which we will call a clock). As the clock tends to infinity, we show that the initial process converges towards a new penalized process, which generally depends on the chosen clock. However, unlike with deterministic clocks, no specific assumptions are needed on the resolvent of the diffusion. We then give a path interpretation of these penalized processes via some universal $\sigma$-finite measures.
\end{abstract}

\section{Introduction.}

\subsection{Penalizations.}

The systematic studies of penalizations started in 2003 with the works of Roynette, Vallois and Yor; see for instance [15], [14] for early papers, and [16] for a monograph on this subject. The starting point of our study is the following classical penalization result: if $|B|$ is a standard reflected Brownian motion with local time at 0 denoted by $L$, then, for any positive integrable function $f$ and any bounded adapted (with respect to the filtration of $|B|)$ process $\left(F_{t}\right)$,

$$
\lim _{t^{\prime} \rightarrow+\infty} \frac{\mathbb{P}\left[F_{t} f\left(L_{t^{\prime}}\right)\right]}{\mathbb{P}\left[f\left(L_{t^{\prime}}\right)\right]}=\mathbb{P}\left[F_{t} \frac{M_{t}}{M_{0}}\right]=: \mathbb{Q}\left[F_{t}\right]
$$

where $M$ is the classical Azéma-Yor martingale (see [1]):

$$
M_{t}=f\left(L_{t}\right)\left|B_{t}\right|+\int_{0}^{+\infty} f\left(x+L_{t}\right) \mathrm{d} x, \quad t \geq 0 .
$$

Then, under the new penalized measure $\mathbb{Q}$, the random variable $L_{\infty}$ is finite and the penalized process is seen to be transient. In fact, its paths may roughly be described as the concatenation of a weighted reflected Brownian bridge and a three-dimensional Bessel process; see [14].

2010 Mathematics Subject Classification. Primary 60F05; Secondary 60J60, 60G44.

Key Words and Phrases. penalization, one-dimensional diffusion, local time, excursion measure.

The first and the second authors were supported by JSPS-MAEDI Sakura program. The second author was supported by MEXT KAKENHI grant 26800058, 24540390 and 15H03624. The third author was supported by MEXT KAKENHI grant 23740073. 
This classical example on the reflected Brownian motion was generalized to many other processes: we may refer in particular to Debs [5] for random walks, Najnudel, Roynette and Yor [10] for Markov chains and Bessel processes, Yano, Yano and Yor [20] for stable processes, or Salminen and Vallois [17] and Profeta [11], [12] for linear diffusions. In most of these papers, a (sometimes implicit but rather strong) condition is made on the considered process, basically stating that a given quantity is regularly varying. We shall give a short account of this classical setup at the end of this introduction.

In this paper, we shall focus on local time penalizations for generalized linear diffusions, but without any assumption of regular variations. To do so, we shall replace the constant time $t^{\prime}$ by a clock $\tau=\tau_{\lambda}$, i.e., a family of random times parametrized by $\lambda$ in a directed set such that $\tau=\tau_{\lambda}$ tends to $+\infty$ a.s. We will deal, for example, with the exponential clock $\tau=\boldsymbol{e}_{q}:=\boldsymbol{e} / q, q>0$ for an independent standard exponential random variable $\boldsymbol{e}$ and with a parameter $q>0$ equipped with the decreasing direction; in fact, $\boldsymbol{e}_{q} \rightarrow+\infty$ a.s. as $q \downarrow 0$. We shall study limits such as

$$
\lim _{\tau \rightarrow+\infty} \frac{\mathbb{P}\left[F_{t} f\left(L_{\tau}\right)\right]}{\mathbb{P}\left[f\left(L_{\tau}\right)\right]}
$$

(the limit here is taken along $\tau=\tau_{\lambda}$ with respect to the parameter $\lambda$, and it can also be understood in the sense of Moore-Smith convergence) and prove in particular that

i) no conditions are needed on the characteristics of the diffusion for the existence of a limit,

ii) the limit depends, in general, of the chosen clock.

Examples of such results already appear in the literature, dealing with processes conditioned to avoid 0, i.e. with the function $f(u)=1_{\{u=0\}}$. We refer to Knight [8] for Brownian motions, Chaumont and Doney [4] and Doney [4] for Lévy processes, and Yano and Yano $[\mathbf{1 9}]$ for diffusions.

\subsection{Notations.}

We start with some notations which are borrowed from [19]. Let us consider a generalized one-dimensional diffusion $X$ (in the sense of Watanabe [18]) defined on an interval $I$ whose left boundary is 0 , with scale function $s(x)=x$ and speed measure $\mathrm{d} m(x)$. We assume that the function $m:[0,+\infty) \rightarrow[0,+\infty]$ is non-decreasing, rightcontinuous, null at 0 and such that

$$
m \text { is }\left\{\begin{array}{l}
\text { strictly increasing on }\left[0, \ell^{\prime}\right), \\
\text { flat and finite on }\left[\ell^{\prime}, \ell\right), \\
\text { infinite on }[\ell,+\infty)
\end{array}\right.
$$

where $0<\ell^{\prime} \leq \ell \leq+\infty$. The choice of the right boundary point of $I$ will depend on $m$; see Section 7 for a sum-up of the different situations (as well as some examples), or [19, Section 2] for a detailed explanation. As for the left boundary point, we assume that 0 is regular-reflecting for $X$. This implies in particular that $X$ admits a local time at 0 , which we shall denote $\left(L_{t}, t \geq 0\right)$, normalized so that 


$$
\mathbb{P}_{x}\left[\int_{0}^{\infty} \mathrm{e}^{-q t} \mathrm{~d} L_{t}\right]=r_{q}(x, 0), \quad x \in I^{\prime}
$$

where $r_{q}(x, y)$ denotes the resolvent density of $X$ with respect to $\mathrm{d} m(y)$ and where $I^{\prime}$ is defined in Section 7 (see also [19, Section 2]). From this formula, we easily obtain the Laplace transform of the first hitting time of the level 0 by $X$ :

$$
\mathbb{P}_{x}\left[\mathrm{e}^{-q T_{0}}\right]=\frac{r_{q}(x, 0)}{r_{q}(0,0)}, \quad x \in I^{\prime}
$$

where $T_{0}=\inf \left\{t \geq 0: X_{t}=0\right\}$. The right-continuous inverse $\eta_{u}^{0}=\inf \left\{t \geq 0: L_{t}>u\right\}$ of $L$ is, when considered under $\mathbb{P}_{0}$, a subordinator. We can compute its Laplace exponent easily by using (1.2), as

$$
\mathbb{P}_{x}\left[\mathrm{e}^{-q \eta_{u}^{0}}\right]=\mathrm{e}^{-u / H(q)} \quad \text { with } H(q):=r_{q}(0,0) .
$$

Let $\phi_{q}$ and $\psi_{q}$ be the two classical eigenfunctions associated to $X$ via the integral equations, for $x \in[0, \ell)$ :

$$
\begin{aligned}
& \phi_{q}(x)=1+q \int_{0}^{x} d y \int_{(0, y]} \phi_{q}(z) \mathrm{d} m(z), \\
& \psi_{q}(x)=x+q \int_{0}^{x} d y \int_{(0, y]} \psi_{q}(z) \mathrm{d} m(z) .
\end{aligned}
$$

With these notations, the resolvent density of $X$ is given by

$$
r_{q}(x, y)=r_{q}(y, x)=H(q) \phi_{q}(x)\left(\phi_{q}(y)-\frac{\psi_{q}(y)}{H(q)}\right), \quad 0 \leq x \leq y, \quad x, y \in I^{\prime},
$$

We denote $m(\infty):=\lim _{x \rightarrow+\infty} m(x), \pi_{0}:=1 / m(\infty)$ and

$$
h_{q}(x)=r_{q}(0,0)-r_{q}(0, x) .
$$

We finally define, following $[\mathbf{1 9}]$,

$$
h_{0}(x)=\lim _{q \downarrow 0} h_{q}(x)=x-\pi_{0} \int_{0}^{x} m(y) \mathrm{d} y,
$$

and we call $h_{0}$ the normalized zero resolvent.

\subsection{Main results.}

We now outline the main results of the paper. For simplicity in this introduction, we assume that $\ell^{\prime}=\ell=\infty$ and we take up the following three cases:

\begin{tabular}{|l|l|l|}
\hline Boundary $\infty$ & $m(\infty)$ & $\int_{(1, \infty)} x \mathrm{~d} m(x)$ \\
\hline (i) type-1-natural & $=\infty$ & $=\infty$ \\
\hline (ii) type-2-natural & $<\infty$ & $=\infty$ \\
\hline (iii) entrance & $<\infty$ & $<\infty$ \\
\hline
\end{tabular}


For instance, the Brownian motion reflected at 0 is an example of case (i), where $\pi_{0}=0$ and $h_{0}(x)=x$.

Let $\mathcal{F}_{t}^{0}=\sigma\left(X_{s}: s \leq t\right)$ and $\mathcal{F}_{t}=\mathcal{F}_{t+}^{0}$. Let $\mathcal{L}_{+}^{1}$ denote the set of non-negative functions $f$ on $[0, \infty)$ such that $\int_{0}^{\infty} f(u) \mathrm{d} u<\infty$. For each choice of a clock $\tau=\tau_{\lambda}$ parametrized by $\lambda$ and a function $f \in \mathcal{L}_{+}^{1}$, we shall find a positive function $\rho(\lambda)$, a supermartingale $N^{f}$ and a martingale $M^{f}$ with respect to $\left(\mathcal{F}_{t}\right)$ such that the following convergences hold: for any $t>0$ and any bounded adapted process $\left(F_{t}\right)$, we have

$\rho(\lambda) \mathbb{P}_{x}\left[F_{t} f\left(L_{\tau_{\lambda}}\right) ; t<\tau_{\lambda}\right] \underset{\tau=\tau_{\lambda} \rightarrow \infty}{\longrightarrow} \mathbb{P}_{x}\left[F_{t} N_{t}^{f}\right]$ and $\rho(\lambda) \mathbb{P}_{x}\left[F_{t} f\left(L_{\tau_{\lambda}}\right)\right] \underset{\tau=\tau_{\lambda} \rightarrow \infty}{\longrightarrow} \mathbb{P}_{x}\left[F_{t} M_{t}^{f}\right]$.

From these formulae we can obtain the penalization limits of the form

$$
\frac{\mathbb{P}_{x}\left[F_{t} f\left(L_{\tau}\right) ; t<\tau\right]}{\mathbb{P}_{x}\left[f\left(L_{\tau}\right)\right]} \underset{\tau \rightarrow \infty}{\rightarrow} \mathbb{P}_{x}\left[F_{t} \frac{N_{t}^{f}}{N_{0}^{f}}\right] \text { and } \frac{\mathbb{P}_{x}\left[F_{t} f\left(L_{\tau}\right)\right]}{\mathbb{P}_{x}\left[f\left(L_{\tau}\right)\right]} \underset{\tau \rightarrow \infty}{\longrightarrow} \mathbb{P}_{x}\left[F_{t} \frac{M_{t}^{f}}{M_{0}^{f}}\right]
$$

which allow to construct new (sub-)probabilities. Note that $N_{t}^{f}$ and $M_{t}^{f}$ may differ according to a particular choice of a clock.

We shall study such penalization limits with four different clocks as below.

\subsubsection{The exponential clock (see Theorem 2.4).}

Recall that $\boldsymbol{e}$ denotes a standard exponential random variable which is independent of the considered diffusion $X$ and $\boldsymbol{e}_{q}=\boldsymbol{e} / q$ with a parameter $q>0$. We may adopt $\left\{\boldsymbol{e}_{q}: q>0\right\}$ as a clock since $\boldsymbol{e}_{q} \rightarrow \infty$ a.s. as $q \downarrow 0$.

Here we assume for notational simplicity that $\boldsymbol{e}$ is defined on the same probability space as $X$; in particular, the expectations are taken on $X$ and $\boldsymbol{e}_{q}=\boldsymbol{e} / q$ at the same time. Note that $\boldsymbol{e}_{q}$ is independent of $\sigma\left(\bigcup_{t} \mathcal{F}_{t}\right)$ because the filtration $\left(\mathcal{F}_{t}\right)$ is generated by $X$.

TheOrem 1.1. Let $f \in \mathcal{L}_{+}^{1}$ and $x \geq 0$. For any $t>0$ and any bounded adapted process $\left(F_{t}\right)$,

$H(q) \mathbb{P}_{x}\left[F_{t} f\left(L_{\boldsymbol{e}_{q}}\right) ; t<\boldsymbol{e}_{q}\right] \underset{q \downarrow 0}{\longrightarrow} \mathbb{P}_{x}\left[F_{t} N_{t}^{h_{0}, f}\right] \quad$ and $H(q) \mathbb{P}_{x}\left[F_{t} f\left(L_{\boldsymbol{e}_{q}}\right)\right] \underset{q \downarrow 0}{\longrightarrow} \mathbb{P}_{x}\left[F_{t} M_{t}^{h_{0}, f}\right]$

where the $\mathbb{P}_{x}$-supermartingale $N^{h_{0}, f}$ and the $\mathbb{P}_{x}$-martingale $M^{h_{0}, f}$ are defined by

$$
N_{t}^{h_{0}, f}=h_{0}\left(X_{t}\right) f\left(L_{t}\right)+\int_{0}^{+\infty} f\left(L_{t}+u\right) \mathrm{d} u, \quad t \geq 0
$$

and

$$
M_{t}^{h_{0}, f}=N_{t}^{h_{0}, f}+\pi_{0} \int_{0}^{t} f\left(L_{u}\right) \mathrm{d} u, \quad t \geq 0 .
$$

\subsubsection{The hitting time clock (see Theorem 3.3).}

For $a \in I$, let $T_{a}=\inf \left\{t \geq 0: X_{t}=a\right\}$ denote the first hitting time of $a$ by $X$. We may adopt $\left\{T_{a}: a \geq 0\right\}$ as a clock since $T_{a} \rightarrow \infty$ a.s. as $a \rightarrow \infty$. 
TheOrem 1.2. Assume that $\infty$ is natural. Let $f \in \mathcal{L}_{+}^{1}$ and $x \geq 0$. For any $t>0$ and any bounded adapted process $\left(F_{t}\right)$,

$$
a \mathbb{P}_{x}\left[F_{t} f\left(L_{T_{a}}\right) ; t<T_{a}\right] \underset{a \uparrow+\infty}{\longrightarrow} \mathbb{P}_{x}\left[F_{t} M_{t}^{s, f}\right] \quad \text { and } \quad a \mathbb{P}_{x}\left[F_{t} f\left(L_{T_{a}}\right)\right] \underset{a \uparrow+\infty}{\longrightarrow} \mathbb{P}_{x}\left[F_{t} M_{t}^{s, f}\right]
$$

where $M^{s, f}$ is the $\mathbb{P}_{x}$-martingale defined by

$$
M_{t}^{s, f}=X_{t} f\left(L_{t}\right)+\int_{0}^{+\infty} f\left(L_{t}+u\right) \mathrm{d} u, \quad t \geq 0 .
$$

(Here by the superscript $s$ we mean the scale function $s(x)=x$.)

\subsubsection{The inverse local time clock (see Theorems 4.5 and 4.7).}

For $a \geq 0$, let $\left(L_{t}^{a}, t \geq 0\right)$ denote the local time of $X$ at level $a$, and define its right-continuous inverse:

$$
\eta_{u}^{a}=\inf \left\{t \geq 0, L_{t}^{a}>u\right\}
$$

We may adopt as a clock $\left\{\eta_{u}^{a}: a \geq 0\right\}$ for a fixed $u>0$, since $\eta_{u}^{a} \geq T_{a} \rightarrow \infty$ a.s. as $a \rightarrow \infty$.

Theorem 1.3. Assume that $\infty$ is natural. Let $f \in \mathcal{L}_{+}^{1}, x \geq 0$ and $u>0$. For any $t>0$ and any bounded adapted process $\left(F_{t}\right)$,

$$
a \mathbb{P}_{x}\left[F_{t} f\left(L_{\eta_{u}^{a}}\right) ; t<\eta_{u}^{a}\right] \underset{a \uparrow+\infty}{\longrightarrow} \mathbb{P}_{x}\left[F_{t} M_{t}^{s, f}\right] \quad \text { and } \quad a \mathbb{P}_{x}\left[F_{t} f\left(L_{\eta_{u}^{a}}\right)\right] \underset{a \uparrow+\infty}{\longrightarrow} \mathbb{P}_{x}\left[F_{t} M_{t}^{s, f}\right]
$$

where $M^{s, f}$ is the $\mathbb{P}_{x}$-martingale defined above.

We obtain here the same penalization limit as that of Theorem 1.2. In spite of this fact, the proofs of the two theorems are quite different; we know that $\eta_{u}^{a} \stackrel{\text { law }}{=} T_{a}+\widetilde{\eta}_{u}^{a}$ where $\widetilde{\eta}_{u}^{a}$ is the inverse local time at $a$ of (an independent copy of) $X$ started at $a$ but this fact cannot reduce Theorem 1.3 to Theorem 1.2.

We may also adopt as a clock $\left\{\eta_{u}^{a}: u \geq 0\right\}$ for a fixed $a>0$, since $\eta_{u}^{a} \rightarrow \infty$ a.s. as $u \rightarrow \infty$. For this clock we only consider the weights $f\left(L_{\eta_{u}^{a}}\right)$ for $f(u)=\mathrm{e}^{-\beta u}$.

TheOREm 1.4. Let $x, a \geq 0$ and $\beta>0$. For $t>0$ and any bounded adapted process $\left(F_{t}\right)$,

$$
\mathrm{e}^{\beta u /(1+a \beta)} \mathbb{P}_{x}\left[F_{t} \mathrm{e}^{-\beta L_{\eta_{u}^{a}}} ; t<\eta_{u}^{a}\right] \underset{u \uparrow+\infty}{\longrightarrow} \mathbb{P}_{x}\left[F_{t} M_{t}^{\beta, a}\right]
$$

and

$$
\mathrm{e}^{\beta u /(1+a \beta)} \mathbb{P}_{x}\left[F_{t} \mathrm{e}^{-\beta L_{\eta_{u} a}}\right] \underset{u \uparrow+\infty}{\longrightarrow} \mathbb{P}_{x}\left[F_{t} M_{t}^{\beta, a}\right]
$$

where $M^{\beta, a}$ is the $\mathbb{P}_{x}$-martingale defined by 


$$
M_{t}^{\beta, a}=\frac{1+\beta\left(X_{t} \wedge a\right)}{1+\beta a} \exp \left(-\beta L_{t}+\frac{\beta}{1+\beta a} L_{t}^{a}\right), \quad t \geq 0 .
$$

\subsection{Comparison among the penalized measures.}

Let $f \in \mathcal{L}_{+}^{1}$. We denote by $\mathbb{Q}_{x}^{h_{0}, f}$ the probability measure such that for any $t>0$,

$$
\mathbb{Q}_{x}^{h_{0}, f}(A)=\mathbb{P}_{x}\left[1_{A} \frac{M_{t}^{h_{0}, f}}{M_{0}^{h_{0}, f}}\right], \quad A \in \mathcal{F}_{t} .
$$

We also define $\mathbb{Q}_{x}^{s, f}$ (resp. $\mathbb{Q}_{x}^{\beta, a}$ ) by replacing $M^{h_{0}, f}$ by $M^{s, f}$ (resp. $M^{\beta, a}$ ); when we speak of $M^{s, f}$ or $\mathbb{Q}_{x}^{s, f}$ we always assume $\infty$ is natural. Let us compare these three measures. We assume for simplicity that $\int_{0}^{\infty} f(u) \mathrm{d} u=1$ and we notice that $M_{0}^{h, f}=N_{0}^{h, f}=1$ for both $h=h_{0}$ and $h=s$.

When $\pi_{0}=0$, we have $h_{0}=s$ and hence the processes $M^{h_{0}, f}$ and $M^{s, f}$ agree, which implies $\mathbb{Q}_{x}^{h_{0}, f}=\mathbb{Q}_{x}^{s, f}$. Therefore, in this specific situation, Theorems 1.1, 1.2 and 1.3 yield the same penalized process. This is for instance the case for the reflected Brownian motion. When $\pi_{0}>0$, i.e., 0 is positive recurrent, the two processes $M^{h_{0}, f}$ and $M^{s, f}$ disagree; they can be considered to be different generalizations of the Azéma-Yor martingales.

Let us focus on the laws of the total local time $L_{\infty}$. The aim of the penalization procedure was to reduce the local time of the original process, so we shall check, for each case, if the strength of the penalization was strong enough to make the total local time of the penalized process finite. For simplicity we only consider the case $x=0$. For $u \geq 0$, from the optional stopping theorem,

$$
\mathbb{Q}_{0}^{h_{0}, f}\left(L_{t} \geq u\right)=\mathbb{P}_{0}\left[M_{t}^{h_{0}, f} 1_{\left\{L_{t} \geq u\right\}}\right]=\mathbb{P}_{0}\left[M_{\eta_{u}^{0}}^{h_{0}, f} 1_{\left\{\eta_{u}^{0} \leq t\right\}}\right],
$$

and then from the monotone convergence theorem,

$$
\mathbb{Q}_{0}^{h_{0}, f}\left(L_{\infty} \geq u\right)=\lim _{t \rightarrow \infty} \mathbb{Q}_{0}^{h_{0}, f}\left(L_{t} \geq u\right)=\mathbb{P}_{0}\left[M_{\eta_{u}^{0}}^{h_{0}, f}\right]
$$

By definition of $M^{h_{0}, f}$, since $X_{\eta_{u}^{0}}=0$ and since $L_{\eta_{u}^{0}}=u$,

$$
\mathbb{P}_{0}\left[M_{\eta_{u}^{0}}^{h_{0}, f}\right]=\int_{u}^{+\infty} f(y) \mathrm{d} y+\pi_{0} \mathbb{P}_{0}\left[\int_{0}^{\eta_{u}^{0}} f\left(L_{r}\right) \mathrm{d} r\right]
$$

By the change of variables $r=\eta_{s}^{0}$ and since $\mathbb{P}_{0}\left[\eta_{u}^{0}\right]=m(\infty) u$,

$$
\pi_{0} \mathbb{P}_{0}\left[\int_{0}^{\eta_{u}^{0}} f\left(L_{r}\right) \mathrm{d} r\right]=\pi_{0} \mathbb{P}_{0}\left[\int_{0}^{u} f(s) \mathrm{d} \eta_{s}^{0}\right]=\pi_{0} m(\infty) \int_{0}^{u} f(s) \mathrm{d} s .
$$

We therefore deduce that

$$
\begin{cases}\mathbb{Q}_{0}^{h_{0}, f}\left(L_{\infty} \in \mathrm{d} u\right)=f(u) \mathrm{d} u & \left(\text { if } \pi_{0}=0\right), \\ \mathbb{Q}_{0}^{h_{0}, f}\left(L_{\infty}=\infty\right)=1 & \left(\text { if } \pi_{0}>0\right) .\end{cases}
$$


In other words, when $\pi_{0}=0$, we went from an original process spending an infinite amount of time at 0 to a penalized process whose total local time at 0 is finite. A similar analysis shows that:

$$
\mathbb{Q}_{0}^{s, f}\left(L_{\infty} \in \mathrm{d} u\right)=f(u) \mathrm{d} u \quad\left(\text { in any case } \pi_{0}=0 \text { or } \pi_{0}>0\right) .
$$

By the same argument as (1.10)-(1.11), we can obtain

$$
\mathbb{Q}_{0}^{\beta, a}\left(L_{\infty} \geq u\right)=\mathbb{P}_{0}\left[\frac{M_{\eta_{u}^{0}}^{\beta, a}}{M_{0}^{\beta, a}}\right]=\exp (-\beta u) \mathbb{P}_{0}\left[\exp \left(\frac{\beta}{1+\beta a} L_{\eta_{u}^{0}}^{a}\right)\right]
$$

Then, by a direct adaptation of the proof of Lemma 4.1 and by analytic continuation, we obtain

$$
\mathbb{P}_{0}\left[\exp \left(\lambda L_{\eta_{u}^{0}}^{a}\right)\right]=\exp \left(\frac{\lambda u}{1-\lambda a}\right), \quad \lambda<1 / a .
$$

Hence we deduce that $\mathbb{Q}_{0}^{\beta, a}\left(L_{\infty} \geq u\right)=1$ for all $u>0$, from which we conclude that

$$
\mathbb{Q}_{0}^{\beta, a}\left(L_{\infty}=\infty\right)=1 \quad\left(\text { in any case } \pi_{0}=0 \text { or } \pi_{0}>0\right) .
$$

Finally, we see that the three cases we have studied provide three different behaviors:

(i) $\mathbb{Q}_{0}^{s, f}$ is a strong penalization: $\mathbb{Q}_{0}^{s, f}\left(L_{\infty}<\infty\right)=1$ whatever the case.

(ii) $\mathbb{Q}_{0}^{h_{0}, f}$ is an intermediate penalization: $\mathbb{Q}_{0}^{h_{0}, f}\left(L_{\infty}<\infty\right)=1$ or 0 according to $\pi_{0}$.

(iii) $\mathbb{Q}_{0}^{\beta, a}$ is a weak penalization: $\mathbb{Q}_{0}^{\beta, a}\left(L_{\infty}<\infty\right)=0$ whatever the case.

\subsection{The case of a constant clock.}

One of the most interesting features of the previous theorems is their universality: the results we obtain are the same for any diffusions, and no conditions are imposed on their characteristics. Such universality is no longer true for constant clocks.

Assume for instance that the diffusion $X$ is recurrent, with $\infty$ a natural boundary. Then, to get the convergence

$$
\frac{\mathbb{P}_{x}\left[F_{t} f\left(L_{t^{\prime}}\right)\right]}{\mathbb{P}_{x}\left[f\left(L_{t^{\prime}}\right)\right]} \underset{t^{\prime} \rightarrow \infty}{\longrightarrow} \mathbb{P}_{x}\left[F_{t} \frac{M_{t}^{s, f}}{M_{0}^{s, f}}\right]
$$

like in the reflected Brownian case (with $M^{s, f}$ given by (1.9)), one needs to add an extra assumption. A sufficient condition is given by Salminen-Vallois [17], who require that

the (normalized) Lévy measure $\nu$ of the subordinator $\eta^{0}$ be subexponential.

It may be shown that (1.13) holds for instance if the function $1 /(q H(q))$ is regularly varying at $q \rightarrow 0^{+}$with exponent in $(0,1)$. We refer here to Profeta [11], where other conditions are also discussed. 
If such extra assumptions are not fulfilled, then generally the martingale we obtained is different and the asymptotics of $\mathbb{P}_{x}\left[f\left(L_{t^{\prime}}\right)\right]$ depends on the function $f$ (as in Theorem 1.4). We give below two examples.

(i) Take to simplify $f(u)=1_{\{u=0\}}$. Then, under Salminen-Vallois' condition (1.13),

$$
\mathbb{P}_{x}\left(T_{0}>t^{\prime}\right) \underset{t^{\prime} \rightarrow \infty}{\sim} x \nu\left(\left(t^{\prime},+\infty\right)\right) \text { and } \frac{\mathbb{P}_{x}\left[F_{t} 1_{\left\{T_{0}>t^{\prime}\right\}}\right]}{\mathbb{P}_{x}\left(T_{0}>t^{\prime}\right)} \underset{t^{\prime} \rightarrow \infty}{\longrightarrow} \mathbb{P}_{x}\left[F_{t} \frac{X_{t}}{x} 1_{\left\{T_{0}>t\right\}}\right]
$$

which is a special instance of (1.12). If we now consider an Ornstein-Uhlenbeck process $Z$ with parameter $\gamma>0$, for which their condition (1.13) on $\nu$ does not hold since for $\varepsilon$ small enough

$$
\left.\lim _{x \rightarrow+\infty} e^{\varepsilon x} \nu(x,+\infty)=0 \quad \text { (instead of }+\infty\right),
$$

then

$$
\mathbb{P}_{x}\left(T_{0}>t^{\prime}\right) \underset{t^{\prime} \rightarrow \infty}{\sim} 2 x \sqrt{\frac{\gamma}{\pi}} e^{-\gamma t^{\prime}} \text { and } \frac{\mathbb{P}_{x}\left[F_{t} 1_{\left\{T_{0}>t^{\prime}\right\}}\right]}{\mathbb{P}_{x}\left(T_{0}>t^{\prime}\right)} \underset{t^{\prime} \rightarrow \infty}{\longrightarrow} \mathbb{P}_{x}\left[F_{t} \frac{Z_{t}}{x} e^{\gamma t} 1_{\left\{T_{0}>t\right\}}\right],
$$

in which case the structure of the martingale is different.

(ii) Assume now that $X$ is a Brownian motion reflected on $[0,1]$ and take $f(u)=e^{-\alpha u}$ with $\alpha>0$. Then

$$
\mathbb{P}_{x}\left[e^{-\alpha L_{t^{\prime}}} \underset{t^{\prime} \rightarrow \infty}{\sim} \kappa e^{-r^{2} t^{\prime}} \cos (r \sqrt{2}(1-x))\right.
$$

where $r$ is defined as the unique solution in $(0, \pi /(2 \sqrt{2}))$ of the equation $\alpha=$ $r \sqrt{2} \tan (r \sqrt{2})$, and $\kappa$ is an explicit constant. In this case, the asymptotics depend on the chosen weight, i.e. on $\alpha$ here. This yields thus a martingale different from $(1.9)$

$$
\frac{\mathbb{P}_{x}\left[F_{t} e^{-\alpha L_{t^{\prime}}}\right]}{\mathbb{P}_{x}\left[e^{-\alpha L_{t^{\prime}}}\right]} \underset{t^{\prime} \rightarrow \infty}{\longrightarrow} \mathbb{P}_{x}\left[F_{t} e^{r^{2} t-\alpha L_{t}} \frac{\cos \left(r \sqrt{2}\left(1-X_{t}\right)\right)}{\cos (r \sqrt{2}(1-x))}\right] .
$$

Such a result may be generalized to other reflected diffusions on $[0,1]$, under the assumption that the analytic continuation of $H(q)$ is smaller (at infinity) than a negative power of $|q|$ on a given strip on the complex plane, see Profeta [12].

\subsection{Organization.}

The remaining of this paper is organized as follows. The local time penalizations are studied with an independent exponential clock in Section 2, then with a hitting time clock in Section 3 and finally with inverse local time clocks in Section 4. In Section 5, we discuss some features of the penalized processes, and give a path decomposition via some universal $\sigma$-finite measures. In Section 6, we characterize the limit measure for an exponential weight, in which case the penalized process remains a generalized diffusion. The final section, Section 7, is an appendix on our boundary classification, with some examples. 


\section{Local time penalization with an exponential clock.}

The proof of Theorem 1.1 will be decomposed in three parts. We shall first study the law of $L_{\boldsymbol{e}_{q}}$, then obtain some a.s. convergence results, and finally extend them to get the $\mathcal{L}^{1}$ convergence. Once the law of $L_{\boldsymbol{e}_{q}}$ has been computed via the excursion theory, the method consists in decomposing $\mathbb{P}_{x}\left[f\left(L_{\boldsymbol{e}_{q}}\right) \mid \mathcal{F}_{t}\right]$ according to $\left\{t<\boldsymbol{e}_{q}\right\}$ or $\left\{t \geq \boldsymbol{e}_{q}\right\}$, and then studying both limits separately.

\subsection{The law of $L_{e_{q}}$.}

We start by computing $\mathbb{P}_{x}\left[f\left(L_{\boldsymbol{e}_{q}}\right)\right]$.

Lemma 2.1. Let $f$ be a non-negative measurable function. Let $q>0$ and $x \in I$. Then

$$
\mathbb{P}_{x}\left[f\left(L_{\boldsymbol{e}_{q}}\right)\right]=\frac{1}{H(q)}\left\{h_{q}(x) f(0)+\frac{r_{q}(x, 0)}{r_{q}(0,0)} \int_{0}^{\infty} \mathrm{e}^{-u / H(q)} f(u) \mathrm{d} u\right\} .
$$

Proof. Using the excursion theory, we have, when starting the diffusion at 0 :

$$
\begin{aligned}
\mathbb{P}_{0}\left[\int_{0}^{\infty} f\left(L_{t}\right) q \mathrm{e}^{-q t} \mathrm{~d} t\right] & =\mathbb{P}_{0}\left[\sum_{u} \int_{\eta_{u-}^{0}}^{\eta_{u}^{0}} f(u) q \mathrm{e}^{-q t} \mathrm{~d} t\right] \\
& =\mathbb{P}_{0}\left[\sum_{u} f(u) \mathrm{e}^{-q \eta_{u-}^{0}}\left(1-\mathrm{e}^{-q T_{0}(p(u))}\right)\right]
\end{aligned}
$$

where $\eta_{u}^{0}$ denotes the inverse local time defined around (1.4), $p$ the excursion point process and $T_{0}(p(s))=\eta_{u}^{0}-\eta_{u-}^{0}$ the length of the excursion of $p(s)$. Now, denoting by $\boldsymbol{n}$ the Itô's excursion measure, we have

$$
\boldsymbol{n}\left[1-\mathrm{e}^{-q T_{0}}\right]=\frac{1}{H(q)},
$$

which follows from (1.4) and the formula $\eta_{u}^{0}=\sum_{s \leq u} T_{0}(p(s))$. Using the Master Formula (see [13, p.475]), we further obtain:

$$
\begin{aligned}
\mathbb{P}_{0}\left[\int_{0}^{\infty} f\left(L_{t}\right) q \mathrm{e}^{-q t} \mathrm{~d} t\right] & =\mathbb{P}_{0}\left[\int_{0}^{\infty} f(u) \mathrm{e}^{-q \eta_{u}^{0}} \mathrm{~d} u\right] \boldsymbol{n}\left[1-\mathrm{e}^{-q T_{0}}\right] \\
& =\int_{0}^{\infty} f(u) \mathrm{e}^{-u / H(q)} \mathrm{d} u \cdot \frac{1}{H(q)}
\end{aligned}
$$

Here we used (1.4) and (2.2). The Markov property then yields the announced result for any starting point $x \in I$ :

$$
\begin{aligned}
\mathbb{P}_{x}\left[f\left(L_{\boldsymbol{e}_{q}}\right)\right] & =\mathbb{P}_{x}\left[\int_{0}^{\infty} f\left(L_{t}\right) q \mathrm{e}^{-q t} \mathrm{~d} t\right] \\
& =\mathbb{P}_{x}\left[\int_{0}^{T_{0}} f\left(L_{t}\right) q \mathrm{e}^{-q t} \mathrm{~d} t\right]+\mathbb{P}_{x}\left[\mathrm{e}^{-q T_{0}}\right] \mathbb{P}_{0}\left[\int_{0}^{\infty} f\left(L_{t}\right) q \mathrm{e}^{-q t} \mathrm{~d} t\right]
\end{aligned}
$$




$$
=f(0)\left\{1-\frac{r_{q}(x, 0)}{r_{q}(0,0)}\right\}+\frac{r_{q}(x, 0)}{r_{q}(0,0)} \cdot \int_{0}^{\infty} f(u) \mathrm{e}^{-u / H(q)} \mathrm{d} u \cdot \frac{1}{H(q)} .
$$

where we used (1.3) and the fact that $L_{t}=0$ for $t \leq T_{0}$.

Letting $q \downarrow 0$, we then deduce the following formulae in the transient and positive recurrent cases. (Note that in the null recurrent case, the limit equals $+\infty$ ).

THEOREM 2.2. Let $f$ be a non-negative measurable function and let $x \in I$. Then the following assertions hold:

(i) If $\ell<\infty$, i.e., 0 is transient, then

$$
\mathbb{P}_{x}\left[f\left(L_{\infty}\right)\right]=\frac{1}{\ell}\left\{x f(0)+\left(1-\frac{x}{\ell}\right) \int_{0}^{\infty} \mathrm{e}^{-u / \ell} f(u) \mathrm{d} u\right\} .
$$

(ii) If $\pi_{0}>0$, i.e., 0 is positive recurrent, then

$$
\mathbb{P}_{x}\left[\int_{0}^{\infty} f\left(L_{t}\right) \mathrm{d} t\right]=\frac{1}{\pi_{0}}\left\{h_{0}(x) f(0)+\int_{0}^{\infty} f(u) \mathrm{d} u\right\}
$$

Proof. (i) We first suppose that $f$ is bounded and set $g=\sup \left\{t: X_{t}=0\right\}$. We see that, almost surely, $f\left(L_{\boldsymbol{e}_{q}}\right)=f\left(L_{g}\right)=f\left(L_{\infty}\right)$ for $q>0$ small enough. Thus

$$
\mathbb{P}_{x}\left[f\left(L_{\boldsymbol{e}_{q}}\right)\right] \underset{q \downarrow 0}{\longrightarrow} \mathbb{P}_{x}\left[f\left(L_{\infty}\right)\right]
$$

by the dominated convergence theorem (here we do not need continuity of $f$ ). Equation (2.3) then follows by letting $q \downarrow 0$ in (2.1). To remove the boundedness assumption, it remains to apply Equation (2.3) with $f \wedge n$ and then let $n \rightarrow \infty$.

(ii) We may rewrite $(2.1)$ as

$$
\mathbb{P}_{x}\left[\int_{0}^{\infty} f\left(L_{t}\right) \mathrm{e}^{-q t} \mathrm{~d} t\right]=\frac{1}{q H(q)}\left\{h_{q}(x) f(0)+\frac{r_{q}(x, 0)}{r_{q}(0,0)} \int_{0}^{\infty} \mathrm{e}^{-u / H(q)} f(u) \mathrm{d} u\right\} .
$$

Equation (2.4) then follows by letting $q \downarrow 0$ and applying the monotone convergence theorem.

\subsection{A.s. convergence for the exponential clock.}

Recall that $\boldsymbol{e}_{q}$ is independent of $\sigma\left(\bigcup_{t} \mathcal{F}_{t}\right)$.

Lemma 2.3. Let $f \in \mathcal{L}_{+}^{1}$ and $x \in I$. For $q>0$, set

$$
N_{t}^{q}=H(q) \mathbb{P}_{x}\left[f\left(L_{\boldsymbol{e}_{q}}\right) 1_{\left\{t<\boldsymbol{e}_{q}\right\}} \mid \mathcal{F}_{t}\right], \quad M_{t}^{q}=H(q) \mathbb{P}_{x}\left[f\left(L_{\boldsymbol{e}_{q}}\right) \mid \mathcal{F}_{t}\right]
$$

and set

$$
\begin{aligned}
& N_{t}^{h_{0}, f}=h_{0}\left(X_{t}\right) f\left(L_{t}\right)+\left(1-\frac{X_{t}}{\ell}\right) \int_{0}^{\infty} \mathrm{e}^{-u / \ell} f\left(L_{t}+u\right) \mathrm{d} u, \\
& M_{t}^{h_{0}, f}=N_{t}^{h_{0}, f}+A_{t}^{h_{0}, f}
\end{aligned}
$$




$$
A_{t}^{h_{0}, f}=\pi_{0} \int_{0}^{t} f\left(L_{u}\right) \mathrm{d} u
$$

(notice that (1.7) and (1.8) are the special cases for $\ell=\ell^{\prime}=\infty$ of (2.5) and (2.6), respectively). Then the following assertions hold:

(i) $N_{t}^{q} \rightarrow N_{t}^{h_{0}, f}$ and $M_{t}^{q} \rightarrow M_{t}^{h_{0}, f}, \mathbb{P}_{x}$-a.s. as $q \downarrow 0$;

(ii) $\left(N_{t}^{h_{0}, f}\right)$ is a $\mathbb{P}_{x}$-supermartingale.

Proof. In what follows in this section we sometimes write to simplify $N_{t}, M_{t}$ and $A_{t}$ for $N_{t}^{h_{0}, f}, M_{t}^{h_{0}, f}$ and $A_{t}^{h_{0}, f}$, respectively.

(i) Since $\left(L_{t}\right)$ is an additive functional and $\boldsymbol{e}_{q}$ an exponential random variable, we have

$$
\begin{aligned}
N_{t}^{q} & =\left.H(q) \mathrm{e}^{-q t} \mathbb{P}_{X_{t}}\left[f\left(a+L_{\boldsymbol{e}_{q}}\right)\right]\right|_{a=L_{t}} \\
& =\mathrm{e}^{-q t}\left\{h_{q}\left(X_{t}\right) f\left(L_{t}\right)+\frac{r_{q}\left(X_{t}, 0\right)}{r_{q}(0,0)} \int_{0}^{\infty} \mathrm{e}^{-u / H(q)} f\left(L_{t}+u\right) \mathrm{d} u\right\}
\end{aligned}
$$

where we have used Lemma 2.1 with $f(a+\cdot) \in \mathcal{L}_{+}^{1}$. It is now clear that $N_{t}^{q} \underset{q \downarrow 0}{\longrightarrow} N_{t}$, $\mathbb{P}_{x}$-a.s. Since

$$
\begin{aligned}
A_{t}^{q}: & =M_{t}^{q}-N_{t}^{q} \\
& =H(q) \mathbb{P}_{x}\left[f\left(L_{\boldsymbol{e}_{q}}\right) 1_{\left\{\boldsymbol{e}_{q} \leq t\right\}} \mid \mathcal{F}_{t}\right] \\
& =q H(q) \int_{0}^{t} f\left(L_{u}\right) \mathrm{e}^{-q u} \mathrm{~d} u,
\end{aligned}
$$

we obtain $A_{t}^{q} \underset{q \downarrow 0}{\longrightarrow} A_{t}$ and $M_{t}^{q} \underset{q \downarrow 0}{\longrightarrow} M_{t}, \mathbb{P}_{x}$-a.s.

(ii) Since for $s \leq t$ we have $1_{\left\{t<\boldsymbol{e}_{q}\right\}} \leq 1_{\left\{s<\boldsymbol{e}_{q}\right\}}$, we easily see that $\left(N_{t}^{q}\right)$ is a $\mathbb{P}_{x^{-}}$ supermartingale. For $s \leq t$, we apply Fatou's lemma to obtain

$$
\mathbb{P}_{x}\left[N_{t} \mid \mathcal{F}_{s}\right] \leq \liminf _{q \downarrow 0} \mathbb{P}_{x}\left[N_{t}^{q} \mid \mathcal{F}_{s}\right] \leq \liminf _{q \downarrow 0} N_{s}^{q}=N_{s}
$$

which shows that $\left(N_{t}\right)$ is a $\mathbb{P}_{x}$-supermartingale.

\section{3. $\quad \mathcal{L}^{1}$ convergence for the exponential clock.}

Theorem 2.4. Let $f \in \mathcal{L}_{+}^{1}$ and $x \in I$.

(i) For any finite stopping time $T$, there is the $\mathcal{L}^{1}$ convergence

$$
N_{T}^{q} \underset{q \downarrow 0}{\longrightarrow} N_{T}^{h_{0}, f} \quad \text { in } \mathcal{L}^{1}\left(\mathbb{P}_{x}\right) .
$$

Consequently, for any bounded adapted process $\left(F_{t}\right)$, it holds that

$$
\lim _{q \downarrow 0} H(q) \mathbb{P}_{x}\left[F_{T} f\left(L_{\boldsymbol{e}_{q}}\right) ; T<\boldsymbol{e}_{q}\right]=\mathbb{P}_{x}\left[F_{T} N_{T}^{h_{0}, f}\right] .
$$


(ii) Assume furthermore that

$$
\mathbb{P}_{x}\left[\int_{0}^{T} f\left(L_{u}\right) \mathrm{d} u\right]<\infty
$$

Then, we have

$$
M_{T}^{q} \underset{q \downarrow 0}{\longrightarrow} M_{T}^{h_{0}, f} \quad \text { in } \mathcal{L}^{1}\left(\mathbb{P}_{x}\right)
$$

and for any bounded adapted process $\left(F_{t}\right)$, it holds that

$$
\lim _{q \downarrow 0} H(q) \mathbb{P}_{x}\left[F_{T} f\left(L_{\boldsymbol{e}_{q}}\right)\right]=\mathbb{P}_{x}\left[F_{T} M_{T}^{h_{0}, f}\right] .
$$

(iii) Any bounded stopping time satisfies (2.7). In particular,

$$
M_{t}^{h_{0}, f}=h_{0}\left(X_{t}\right) f\left(L_{t}\right)+\left(1-\frac{X_{t}}{\ell}\right) \int_{0}^{\infty} \mathrm{e}^{-u / \ell} f\left(L_{t}+u\right) \mathrm{d} u+\pi_{0} \int_{0}^{t} f\left(L_{u}\right) \mathrm{d} u
$$

is a $\mathbb{P}_{x}$-martingale and the identity $N^{h_{0}, f}=M^{h_{0}, f}-A^{h_{0}, f}$ may be regarded as the Doob-Meyer decomposition of the supermartingale $N^{h_{0}, f}$.

Proof. (i) Observe first by Fatou's lemma that

$$
\mathbb{P}_{x}\left[N_{T}\right] \leq \liminf _{n \rightarrow \infty} \mathbb{P}_{x}\left[N_{T \wedge n}\right] \leq \mathbb{P}_{x}\left[N_{0}\right]<\infty .
$$

Let us compute $N_{T}^{q}$. We have

$$
\begin{aligned}
N_{T}^{q} & =\mathrm{e}^{-q T} h_{q}\left(X_{T}\right) f\left(L_{T}\right)+\mathrm{e}^{-q T} \frac{r_{q}\left(X_{T}, 0\right)}{r_{q}(0,0)} \int_{0}^{\infty} \mathrm{e}^{-u / H(q)} f\left(L_{T}+u\right) \mathrm{d} u \\
& =(\mathrm{I})_{q}+(\mathrm{II})_{q} .
\end{aligned}
$$

We write similarly

$$
\begin{aligned}
N_{T} & =h_{0}\left(X_{T}\right) f\left(L_{T}\right)+\left(1-\frac{X_{T}}{\ell}\right) \int_{0}^{\infty} \mathrm{e}^{-u / \ell} f\left(L_{T}+u\right) \mathrm{d} u \\
& =(\mathrm{I})+(\mathrm{II}) .
\end{aligned}
$$

Since $(\mathrm{II})_{q} \leq \int_{0}^{\infty} f(u) \mathrm{d} u$, we may apply the dominated convergence theorem to obtain $(\mathrm{II})_{q} \rightarrow(\mathrm{II})$ in $\mathcal{L}^{1}\left(\mathbb{P}_{x}\right)$.

If $\pi_{0}=0$, then we have $(\mathrm{I})_{q} \leq X_{T} f\left(L_{T}\right)=h_{0}\left(X_{T}\right) f\left(L_{T}\right) \leq N_{T}$. If $\pi_{0}>0$ and $\ell^{\prime}$ is regular-reflecting, then we have $h_{0}(x) \geq c x$ with $c=h_{0}\left(\ell^{\prime}\right) / \ell^{\prime}>0$, since $h_{0}(x)$ is concave. We now have $(\mathrm{I})_{q} \leq X_{T} f\left(L_{T}\right) \leq c^{-1} h_{0}\left(X_{T}\right) f\left(L_{T}\right) \leq c^{-1} N_{T}$. In both cases, since $\mathbb{P}_{x}\left[N_{T}\right] \leq \mathbb{P}_{x}\left[N_{0}\right]<\infty$, we may apply the dominated convergence theorem to obtain $(\mathrm{I})_{q} \rightarrow(\mathrm{I})$ in $\mathcal{L}^{1}\left(\mathbb{P}_{x}\right)$.

If $\pi_{0}>0$ and $\ell^{\prime}$ is either entrance or natural, we have $(\mathrm{I})_{q} \leq X_{T} f\left(L_{T}\right)$. Since we see by (ii) of Lemma 2.3 that 


$$
\mathbb{P}_{x}\left[X_{T} f\left(L_{T}\right)\right] \leq \mathbb{P}_{x}\left[N_{T}^{s, f}\right] \leq \mathbb{P}_{x}\left[N_{0}^{s, f}\right]=x f(0)+\left(1-\frac{x}{\ell}\right) \int_{0}^{\infty} \mathrm{e}^{-u / \ell} f(u) \mathrm{d} u<\infty
$$

we may apply the dominated convergence theorem to obtain $(\mathrm{I})_{q} \rightarrow(\mathrm{I})$ in $\mathcal{L}^{1}\left(\mathbb{P}_{x}\right)$. Therefore we have obtained the former assertion. For the latter assertion, we have

$$
H(q) \mathbb{P}_{x}\left[F_{T} f\left(L_{\boldsymbol{e}_{q}}\right) ; T<\boldsymbol{e}_{q}\right]=\mathbb{P}_{x}\left[F_{T} N_{T}^{q}\right] \underset{q \downarrow 0}{\longrightarrow} \mathbb{P}_{x}\left[F_{T} N_{T}\right] .
$$

(ii) To prove (2.8), it suffices to observe that by (2.7), we have $\int_{0}^{T} f\left(L_{u}\right) \mathrm{e}^{-q u} \mathrm{~d} u \rightarrow$ $\int_{0}^{T} f\left(L_{u}\right) \mathrm{d} u$ in $\mathcal{L}^{1}\left(\mathbb{P}_{x}\right)$. This shows that $A_{T}^{q} \rightarrow A_{T}$ in $\mathcal{L}^{1}\left(\mathbb{P}_{x}\right)$, which implies $M_{T}^{q} \rightarrow M_{T}$ in $\mathcal{L}^{1}\left(\mathbb{P}_{x}\right)$.

(iii) Since

$$
q^{2} \int_{0}^{\infty} \mathbb{P}_{x}\left[\int_{0}^{t} f\left(L_{u}\right) \mathrm{d} u\right] \mathrm{e}^{-q t} \mathrm{~d} t=\mathbb{P}_{x}\left[\int_{0}^{\infty} f\left(L_{u}\right) q \mathrm{e}^{-q u} \mathrm{~d} u\right]=\mathbb{P}_{x}\left[f\left(L_{\boldsymbol{e}_{q}}\right)\right]<\infty
$$

and since $t \mapsto \mathbb{P}_{x}\left[\int_{0}^{t} f\left(L_{u}\right) \mathrm{d} u\right]$ is increasing, we see that $\mathbb{P}_{x}\left[\int_{0}^{t} f\left(L_{u}\right) \mathrm{d} u\right]<\infty$ for all $t \geq 0$. Thus (2.8) holds for all constant times, which implies that $M^{h_{0}, f}$ is a martingale.

REMARK 2.5. As mentioned in the Introduction, if $\ell^{\prime}$ is type-1-natural, then the identity (2.6) becomes

$$
M_{t}^{h_{0}, f}=X_{t} f\left(L_{t}\right)+\int_{0}^{\infty} f\left(L_{t}+u\right) \mathrm{d} u
$$

which is nothing else but the Azéma-Yor martingale $([\mathbf{1}])$. In this sense we may regard the identity (2.6) as a generalization of the Azéma-Yor martingale. Another generalization will be given in Theorem 3.3.

REMARK 2.6. If we take $f(u)=1_{\{u=0\}}$, we have

$$
M_{t}^{h_{0}, f}=h_{0}\left(X_{t}\right) 1_{\left\{T_{0}>t\right\}}+\pi_{0}\left(T_{0} \wedge t\right) .
$$

In particular, from the identity $\mathbb{P}_{x}\left[M_{0}^{h_{0}, f}\right]=\mathbb{P}_{x}\left[M_{t}^{h_{0}, f}\right]$, we obtain

$$
h_{0}(x)=\mathbb{P}_{x}\left[h_{0}\left(X_{t}\right) ; T_{0}>t\right]+\pi_{0} \mathbb{P}_{x}\left[T_{0} \wedge t\right],
$$

which is nothing else but the first assertion of Theorem 6.4 of [19].

\section{Local time penalization with a hitting time clock.}

In this section we assume that $\ell\left(=\ell^{\prime}\right)$ is either entrance or natural. Since any point in $[0, \ell)$ is accessible but $\ell$ is not, we have

$$
\mathbb{P}_{x}\left(T_{a} \rightarrow \infty \text { as } a \uparrow \ell\right)=1 .
$$

We start by computing the law of $L_{T_{a}}$ using the formula (2.3) for the stopped process at $a$. We then prove a.s. and $\mathcal{L}^{1}$ convergence results upon separating the cases $\left\{t<T_{a}\right\}$ 
and $\left\{t \geq T_{a}\right\}$ and using some estimates on the cumulative distribution function of $T_{a}$.

\subsection{A.s. convergence for hitting times clocks.}

We start by computing the quantity $\mathbb{P}_{x}\left[f\left(L_{T_{a}}\right)\right]$.

Lemma 3.1. Let $f \in \mathcal{L}_{+}^{1}$ and $x \in I$. Then, for any $a \in I$ with $x<a$,

$$
\mathbb{P}_{x}\left[f\left(L_{T_{a}}\right)\right]=\frac{1}{a}\left\{x f(0)+\left(1-\frac{x}{a}\right) \int_{0}^{\infty} \mathrm{e}^{-u / a} f(u) \mathrm{d} u\right\} .
$$

Proof. Let $\mathbb{P}_{x}^{a}$ denote the law of $X_{\cdot \wedge T_{a}}$ under $\mathbb{P}_{x}$. Then we have

$$
\mathbb{P}_{x}\left[f\left(L_{T_{a}}\right)\right]=\mathbb{P}_{x}^{a}\left[f\left(L_{\infty}\right)\right]
$$

Since $\left\{X, \mathbb{P}_{x}^{a}\right\}$ is a diffusion process on $[0, a]$ where $a$ is a regular-absorbing boundary, we may use (i) of Theorem 2.2 and obtain (3.1).

Lemma 3.2. Let $f \in \mathcal{L}_{+}^{1}$ and $x \in I$. For any $a \in I$ with $x<a$, set

$$
N_{t}^{a}=a \mathbb{P}_{x}\left[f\left(L_{T_{a}}\right) 1_{\left\{t<T_{a}\right\}} \mid \mathcal{F}_{t}\right], \quad M_{t}^{a}=a \mathbb{P}_{x}\left[f\left(L_{T_{a}}\right) \mid \mathcal{F}_{t}\right]
$$

and

$$
M_{t}^{s, f}=X_{t} f\left(L_{t}\right)+\left(1-\frac{X_{t}}{\ell}\right) \int_{0}^{\infty} \mathrm{e}^{-u / \ell} f\left(L_{t}+u\right) \mathrm{d} u .
$$

Then the following assertions hold:

(i) $N_{t}^{a} \rightarrow M_{t}^{s, f}$ and $M_{t}^{a} \rightarrow M_{t}^{s, f}, \mathbb{P}_{x}$-a.s. as a $\uparrow \ell$;

(ii) $\left(M_{t}^{s, f}\right)$ is a $\mathbb{P}_{x}$-supermartingale and is a local $\mathbb{P}_{x}$-martingale. $M_{t}^{s, f}$

Proof. In what follows in this section we sometimes write to simplify $M_{t}$ for

(i) Since $f(b+\cdot) \in \mathcal{L}_{+}^{1}$, we have, by Lemma 3.1 ,

$$
\begin{aligned}
N_{t}^{a} & =\left.a \mathbb{P}_{X_{t}}\left[f\left(b+L_{T_{a}}\right)\right]\right|_{b=L_{t}} 1_{\left\{t<T_{a}\right\}} \\
& =\left\{X_{t} f\left(L_{t}\right)+\left(1-\frac{X_{t}}{a}\right) \int_{0}^{\infty} \mathrm{e}^{-u / a} f\left(L_{t}+u\right) \mathrm{d} u\right\} 1_{\left\{t<T_{a}\right\}} .
\end{aligned}
$$

Using that $T_{a} \rightarrow \infty$ as $a \uparrow \ell$, we then deduce that $N_{t}^{a} \rightarrow M_{t}, \mathbb{P}_{x^{-a . s . ~}}$ Set

$$
A_{t}^{a}=M_{t}^{a}-N_{t}^{a}=a f\left(L_{T_{a}}\right) 1_{\left\{T_{a} \leq t\right\}} .
$$

Since $A_{t}^{a} \rightarrow 0, \mathbb{P}_{x}$-a.s., we further obtain that $M_{t}^{a} \rightarrow M_{t}, \mathbb{P}_{x^{-a . s . ~}}$

(ii) In the same way as (ii) of Lemma 2.3 , we can see that $\left(M_{t}\right)$ is a $\mathbb{P}_{x^{-}}$ supermartingale.

It is obvious that $\left(M_{t}^{a}\right)$ is a $\mathbb{P}_{x}$-martingale. Let $\left\{a_{n}\right\}$ be a sequence of $I$ such that $a_{n} \uparrow \ell$. If we take $\sigma_{n}=\inf \left\{t: X_{t}>a_{n}\right\}$, we have $A_{\sigma_{n} \wedge t}^{a}=a f\left(L_{T_{a}}\right) 1_{\left\{T_{a} \leq \sigma_{n} \wedge t\right\}}=0$ for 
any $a>a_{n}$, so that we have $M_{\sigma_{n} \wedge t}^{a} \rightarrow M_{\sigma_{n} \wedge t}$ in $\mathcal{L}^{1}\left(\mathbb{P}_{x}\right)$ as $a \uparrow \ell$. This shows that $\left(M_{t}\right)$ is a local $\mathbb{P}_{x}$-martingale.

\section{2. $\mathcal{L}^{1}$ convergence for hitting times clocks.}

THEOREM 3.3. Let $f \in \mathcal{L}_{+}^{1}$ and $x \in I$.

(i) For any finite stopping time $T$, there is the $\mathcal{L}^{1}$ convergence

$$
N_{T}^{a} \underset{a \uparrow \ell}{\longrightarrow} M_{T}^{s, f} \text { in } \mathcal{L}^{1}\left(\mathbb{P}_{x}\right) .
$$

Consequently, for any bounded adapted process $\left(F_{t}\right)$, it holds that

$$
a \mathbb{P}_{x}\left[F_{T} f\left(L_{T_{a}}\right) ; T<T_{a}\right] \underset{a \uparrow \ell}{\longrightarrow} \mathbb{P}_{x}\left[F_{T} M_{T}^{s, f}\right] .
$$

(ii) Suppose furthermore that $\ell$ is natural. Then, for any $f \in \mathcal{L}_{+}^{1}$ and for any bounded stopping time $T$, we have

$$
M_{T}^{a} \underset{a \uparrow \ell}{\longrightarrow} M_{T}^{s, f} \quad \text { in } \mathcal{L}^{1}\left(\mathbb{P}_{x}\right)
$$

and for any bounded adapted process $\left(F_{t}\right)$, it holds that

$$
a \mathbb{P}_{x}\left[F_{T} f\left(L_{T_{a}}\right)\right] \underset{a \uparrow \ell}{\longrightarrow} \mathbb{P}_{x}\left[F_{T} M_{T}^{s, f}\right] .
$$

In particular, $M^{s, f}$ is a $\mathbb{P}_{x}$-martingale.

Proof. We start with Point (i). We have

$$
\begin{aligned}
& N_{T}^{a}=X_{T} f\left(L_{T}\right) 1_{\left\{T<T_{a}\right\}}+\left(1-\frac{X_{T}}{a}\right) \int_{0}^{\infty} \mathrm{e}^{-u / a} f\left(L_{T}+u\right) \mathrm{d} u 1_{\left\{T<T_{a}\right\}}, \\
& M_{T}=X_{T} f\left(L_{T}\right)+\left(1-\frac{X_{T}}{\ell}\right) \int_{0}^{\infty} \mathrm{e}^{-u / \ell} f\left(L_{T}+u\right) \mathrm{d} u .
\end{aligned}
$$

Since $N_{T}^{a} \leq M_{T}$ and since

$$
\mathbb{P}_{x}\left[M_{T}\right] \leq \liminf _{n \rightarrow \infty} \mathbb{P}_{x}\left[M_{T \wedge n}\right] \leq \mathbb{P}_{x}\left[M_{0}\right]<\infty
$$

we may apply the dominated convergence theorem to obtain (3.3). The remaining assertion is obvious.

To prove Point (ii), we shall rely on the following Lemma:

Lemma 3.4. Suppose that $\ell$ is natural. Then

$$
a \mathbb{P}_{x}\left(T_{a} \leq t\right) \underset{a \uparrow \ell}{\longrightarrow} 0 \text { for all } t \geq 0
$$

Proof. If $\ell<\infty$, i.e., $\ell$ is type-3-natural, then (3.6) is obvious.

Suppose $\ell=\infty$. Since the Laplace transform of $T_{a}$ may be written as a ratio of eigenfunctions, we have 


$$
a \mathbb{P}_{x}\left(T_{a} \leq t\right) \leq a \mathrm{e}^{t} \mathbb{P}_{x}\left[\mathrm{e}^{-T_{a}}\right]=\mathrm{e}^{t} \phi_{1}(x) \cdot \frac{a}{\phi_{1}(a)}
$$

Going back to the integral equation (1.5) of $\phi_{1}$ and using that $\ell=\infty$ is natural, we deduce that

$$
\phi_{1}(a)=1+\int_{0}^{a} \mathrm{~d} x \int_{(0, x]} \phi_{1}(y) \mathrm{d} m(y) \geq \int_{0}^{a} \mathrm{~d} x \int_{(0, x]} \mathrm{d} m(y) \underset{a \uparrow \ell}{\longrightarrow} \infty
$$

and, for $a>1$,

$$
\phi_{1}^{\prime}(a) \geq \int_{(0, a]} \mathrm{d} m(x) \int_{0}^{x} \phi_{1}^{\prime}(y) \mathrm{d} y \geq \phi_{1}^{\prime}(1) \int_{(1, a]} \mathrm{d} m(x) \int_{1}^{x} \mathrm{~d} y \underset{a \uparrow \ell}{\longrightarrow} \infty .
$$

Thus, by the l'Hôpital's rule, we obtain $a / \phi_{1}(a) \rightarrow 0$ as $a \uparrow \ell=\infty$ which yields (3.6).

We now come back to the proof of Point (ii) of Theorem 3.3. Suppose that $f \in \mathcal{L}_{+}^{1}$ is bounded. Since $A_{T}^{a} \rightarrow 0, \mathbb{P}_{x}$-a.s. and since

$$
\mathbb{P}_{x}\left[A_{T}^{a}\right] \leq a\|f\|_{\infty} \mathbb{P}_{x}\left(T_{a} \leq T\right) \underset{a \uparrow \ell}{\longrightarrow} 0
$$

we see that $A_{T}^{a} \rightarrow 0$ in $\mathcal{L}^{1}\left(\mathbb{P}_{x}\right)$. Hence we obtain (3.4) and (3.5) in this special case.

We now see that $\mathbb{P}_{x}\left[M_{t}^{s, f}\right]=\mathbb{P}_{x}\left[M_{0}^{s, f}\right]$, i.e.,

$$
\begin{aligned}
\mathbb{P}_{x} & {\left[X_{t} f\left(L_{t}\right)+\left(1-\frac{X_{t}}{\ell}\right) \int_{0}^{\infty} \mathrm{e}^{-u / \ell} f\left(L_{t}+u\right) \mathrm{d} u\right] } \\
& =x f(0)+\left(1-\frac{x}{\ell}\right) \int_{0}^{\infty} \mathrm{e}^{-u / \ell} f(u) \mathrm{d} u
\end{aligned}
$$

holds for all $t \geq 0$ and all bounded $f \in \mathcal{L}_{+}^{1}$. By considering $f \wedge n$, taking $n \rightarrow \infty$ and applying the monotone convergence theorem, we can drop the boundedness assumption and obtain (3.7) for all $t \geq 0$ and all $f \in \mathcal{L}_{+}^{1}$. By (ii) of Lemma 3.2, we see, for any $f \in \mathcal{L}_{+}^{1}$, that $\left(M_{t}^{s, f}\right)$ is a $\mathbb{P}_{x}$-supermartingale with constant expectation, which turns out to be a $\mathbb{P}_{x}$-martingale.

Let $f \in \mathcal{L}_{+}^{1}$. Since $\left(M_{t}^{s, f}\right)$ is a $\mathbb{P}_{x}$-martingale, we may apply the optional stopping theorem to see that

$$
\begin{aligned}
\mathbb{P}_{x}\left[A_{T}^{a}\right] & =\mathbb{P}_{x}\left[X_{T_{a}} f\left(L_{T_{a}}\right) ; T_{a} \leq T\right] \\
& \leq \mathbb{P}_{x}\left[M_{T_{a}} ; T_{a} \leq T\right] \\
& =\mathbb{P}_{x}\left[M_{T} ; T_{a} \leq T\right] \underset{a \uparrow \ell}{\longrightarrow} 0 .
\end{aligned}
$$

Since $A_{T}^{a} \rightarrow 0, \mathbb{P}_{x}$-a.s., we see that $A_{T}^{a} \rightarrow 0$ in $\mathcal{L}^{1}\left(\mathbb{P}_{x}\right)$. Hence we obtain (3.4) and (3.5) in the general case.

Remark 3.5. Suppose $\ell$ is entrance. We claim that $M=M^{s, f}$ is not a true $\mathbb{P}_{x}$-martingale. Indeed, suppose $M$ were a $\mathbb{P}_{x}$-martingale. On the one hand, we would have 


$$
\mathbb{P}_{x}\left[M_{\eta_{u}^{0}}\right]=\lim _{t \rightarrow \infty} \mathbb{P}_{x}\left[M_{\eta_{u}^{0} \wedge t} 1_{\left\{\eta_{u}^{0} \leq t\right\}}\right]=M_{0}=x f(0)+\int_{0}^{\infty} f(r) \mathrm{d} r .
$$

On the other hand, since $X_{\eta_{u}^{0}}=0$ and $\ell=\infty$, we have

$$
\mathbb{P}_{x}\left[M_{\eta_{u}^{0}}\right]=\int_{u}^{\infty} f(r) \mathrm{d} r \underset{u \rightarrow \infty}{\longrightarrow} 0
$$

which would be a contradiction. Note that in the special case $f(u)=1_{\{u=0\}}$ and $M_{t}^{s, f}=$ $X_{t} 1_{\left\{T_{0}>t\right\}}$ this result has already been obtained in Theorem 6.5 of [19] in other words: $s(x)=x$ is not invariant with respect to the stopped process.

\section{Local time penalization with inverse local time clocks.}

We recall that $\eta_{u}^{a}$ denotes the right-continuous inverse of $\left(L_{t}^{a}, t \geq 0\right)$ :

$$
\eta_{u}^{a}=\inf \left\{t \geq 0, L_{t}^{a}>u\right\}
$$

The proofs given in this Section follow the same pattern as before. The main ingredients are excursion theory away from $a \neq 0$ and some estimates on modified Bessel functions.

\subsection{Limit as $a$ tends to infinity with $u$ being fixed.}

Suppose $\ell^{\prime}(=\ell=\infty)$ is either entrance, type-1-natural or type-2-natural. We thus have, for any $x \in I$ and any $u>0$,

$$
\mathbb{P}_{x}\left(\eta_{u}^{a}<\infty\right)=1 \quad \text { and } \quad \eta_{u}^{a} \geq T_{a} \underset{a \rightarrow \infty}{\longrightarrow} \infty, \mathbb{P}_{x} \text {-a.s. }
$$

in fact, the former is implied by

$$
\mathbb{P}_{x}\left[\mathrm{e}^{-q \eta_{u}^{a}}\right] \underset{q \downarrow 0}{\longrightarrow} 1
$$

since $r_{q}(a, a) \underset{q \downarrow 0}{\longrightarrow} \infty$ and

$$
\mathbb{P}_{x}\left[\mathrm{e}^{-q \eta_{u}^{a}}\right]=\mathbb{P}_{x}\left[\mathrm{e}^{-q T_{a}}\right] \mathbb{P}_{a}\left[\mathrm{e}^{-q \eta_{u}^{a}}\right]=\frac{\phi_{q}(x)}{\phi_{q}(a)} \exp \left(-\frac{u}{r_{q}(a, a)}\right) .
$$

For $\nu \geq 0$, we denote by $I_{\nu}(x)$ the modified Bessel function of the first kind, which may be represented as a series expansion formula (see e.g. [9], Equation (5.7.1) on page 108) by

$$
I_{\nu}(x)=\sum_{n=0}^{\infty} \frac{(x / 2)^{\nu+2 n}}{n ! \Gamma(\nu+n+1)}, \quad x>0 .
$$

We recall the asymptotic formulae (see e.g. [9], Section 5.16):

$$
I_{\nu}(x) \underset{x \downarrow 0}{\sim} \frac{(x / 2)^{\nu}}{\Gamma(1+\nu)}, \quad I_{\nu}(x) \underset{x \rightarrow \infty}{\sim} \frac{\mathrm{e}^{x}}{\sqrt{2 \pi x}} .
$$


Lemma 4.1. Let $a \in(0, \infty)$. Then the process $\left\{\left(L_{\eta_{u}^{a}}\right)_{u \geq 0}, \mathbb{P}_{a}\right\}$ is a compound Poisson process with Laplace transform

$$
\mathbb{P}_{a}\left[\mathrm{e}^{-\beta L_{\eta_{u}^{a}}}\right]=\exp \left\{-u \int_{0}^{\infty}\left(1-\mathrm{e}^{-\beta s}\right) \frac{1}{a^{2}} \mathrm{e}^{-s / a} \mathrm{~d} s\right\}=\mathrm{e}^{-u \beta /(1+\beta a)} .
$$

For any $u>0$ and $f \in \mathcal{L}_{+}^{1}$,

$$
\mathbb{P}_{a}\left[f\left(L_{\eta_{u}^{a}}\right)\right]=\mathrm{e}^{-u / a} f(0)+\int_{0}^{\infty} f(y) \rho_{u}^{a}(y) \mathrm{d} y,
$$

where

$$
\rho_{u}^{a}(y)=\mathrm{e}^{-(u+y) / a} \frac{\sqrt{u / y}}{a} I_{1}\left(\frac{2 \sqrt{u y}}{a}\right) .
$$

Proof. Let $p^{a}(v)$ denote the point process of excursions away from $a$ and $\boldsymbol{n}^{a}$ its excursion measure. Since $L$ increases only on the intervals $\left(\eta_{v-}^{a}, \eta_{v}^{a}\right)$, we have

$$
L_{\eta_{u}^{a}}=\sum_{v \leq u: p^{a}(v) \in\left\{T_{0}<\infty\right\}}\left(L_{\eta_{v}^{a}}-L_{\eta_{v-}^{a}}\right)=\sum_{v \leq u: p^{a}(v) \in\left\{T_{0}<\infty\right\}} L_{T_{a}}\left(p^{a}(v)\right) .
$$

Since $\boldsymbol{n}^{a}\left(T_{0}<T_{a}\right)=1 / a<\infty$, the sum of (4.6) is a finite sum, and so we see that $\left\{\left(L_{\eta_{u}^{a}}\right)_{u \geq 0}, \mathbb{P}_{a}\right\}$ is a compound Poisson process with Lévy measure

$$
\boldsymbol{n}^{a}\left(L_{T_{a}} \in \mathrm{d} s ; T_{0}<T_{a}\right)
$$

By the strong Markov property of $\boldsymbol{n}^{a}$ (see, e.g., [3, Theorem III.3.28]), we have

$$
\boldsymbol{n}^{a}\left(L_{T_{a}}>s ; T_{0}<T_{a}\right)=\boldsymbol{n}^{a}\left(T_{0}<\infty\right) \mathbb{P}_{0}\left(L_{T_{a}}>s\right)=\frac{1}{a} \mathbb{P}_{0}\left(L_{T_{a}}>s\right) .
$$

Let $\lambda_{a}^{0}=\inf \left\{v: p^{0}(v) \in\left\{T_{a}<\infty\right\}\right\}$. Then we have

$$
\mathbb{P}_{0}\left(L_{T_{a}}>s\right)=\mathbb{P}_{0}\left(T_{a}>\eta_{s}^{0}\right)=\mathbb{P}_{0}\left(\lambda_{a}^{0}>s\right)=\mathrm{e}^{-s \boldsymbol{n}^{0}\left(T_{a}<\infty\right)}=\mathrm{e}^{-s / a} .
$$

Thus we obtain (4.4).

Let $\left\{S_{n}\right\}$ be a process with i.i.d. increments $\mathbb{P}\left(S_{n}-S_{n-1}>s\right)=\mathrm{e}^{-s / a}$ such that $S_{0}=0$ and let $N$ be a Poisson variable with mean $u / a$ which is independent of $\left\{S_{n}\right\}$. Then we have $L_{\eta_{u}^{a}} \stackrel{\text { law }}{=} S_{N}$, and hence

$$
\begin{aligned}
\mathbb{P}_{a}\left[f\left(L_{\eta_{u}^{a}}\right)\right] & =\mathbb{P}(N=0) f(0)+\sum_{n=1}^{\infty} \mathbb{P}(N=n) \mathbb{P}\left[f\left(S_{n}\right)\right] \\
& =\mathrm{e}^{-u / a} f(0)+\sum_{n=1}^{\infty} \mathrm{e}^{-u / a} \frac{(u / a)^{n}}{n !} \int_{0}^{\infty} f(y) \frac{(y / a)^{n-1}}{(n-1) !} \mathrm{e}^{-y / a} \frac{\mathrm{d} y}{a} .
\end{aligned}
$$

Thus, using (4.2), we obtain (4.5). 
Lemma 4.2. For $u>0, x, a \in I$ and $f \in \mathcal{L}_{+}^{1}$, it holds that

$$
\begin{aligned}
\mathbb{P}_{x}\left[f\left(L_{\eta_{u}^{a}}\right)\right] & =\frac{x \wedge a}{a} \mathbb{P}_{a}\left[f\left(L_{\eta_{u}^{a}}\right)\right]+\left(1-\frac{x}{a}\right)_{+} \mathbb{P}_{a}\left[f\left(\boldsymbol{e}_{1 / a}+L_{\eta_{u}^{a}}\right)\right] \\
& =\frac{x \wedge a}{a} \mathbb{P}_{a}\left[f\left(L_{\eta_{u}^{a}}\right)\right]+\frac{1}{a}\left(1-\frac{x}{a}\right)_{+} \int_{0}^{\infty} f(y) \tilde{\rho}_{u}^{a}(y) \mathrm{d} y,
\end{aligned}
$$

where

$$
\tilde{\rho}_{u}^{a}(y)=\mathrm{e}^{-(u+y) / a} I_{0}\left(\frac{2 \sqrt{u y}}{a}\right) .
$$

Proof. When $a \leq x$, we have $\mathbb{P}_{x}\left[f\left(L_{\eta_{u}^{a}}\right)\right]=\mathbb{P}_{x}\left[f\left(L_{T_{a}}+L_{\eta_{u}^{a}} \circ \theta_{T_{a}}\right)\right]=\mathbb{P}_{a}\left[f\left(L_{\eta_{u}^{a}}\right)\right]$, which proves identity (4.7).

Suppose $x<a$. Using Lemma 3.1, we have

$$
\begin{aligned}
\mathbb{P}_{x}\left[f\left(L_{\eta_{u}^{a}}\right)\right] & =\mathbb{P}_{x}\left[f\left(L_{T_{a}}+L_{\eta_{u}^{a}} \circ \theta_{T_{a}}\right)\right] \\
& =\frac{x}{a} \mathbb{P}_{a}\left[f\left(L_{\eta_{u}^{a}}\right)\right]+\frac{1}{a}\left(1-\frac{x}{a}\right) \mathbb{P}_{a}\left[\int_{0}^{\infty} \mathrm{e}^{-v / a} f\left(v+L_{\eta_{u}^{a}}\right) \mathrm{d} v\right],
\end{aligned}
$$

which coincides with (4.7). Using the same notation as that of the proof of Lemma 4.1, we obtain

$$
\begin{aligned}
\mathbb{P}_{a}\left[f\left(\boldsymbol{e}_{1 / a}+L_{\eta_{u}^{a}}\right)\right] & =\sum_{n=0}^{\infty} \mathbb{P}(N=n) \mathbb{P}\left[f\left(S_{n+1}\right)\right] \\
& =\sum_{n=0}^{\infty} \mathrm{e}^{-u / a} \frac{(u / a)^{n}}{n !} \int_{0}^{\infty} f(y) \frac{(y / a)^{n}}{n !} \mathrm{e}^{-y / a} \frac{\mathrm{d} y}{a} .
\end{aligned}
$$

Thus, using (4.2), we obtain (4.8).

By (4.3), there exists a constant $C$ such that

$$
\begin{cases}I_{\nu}(x) \leq C x^{\nu} & \text { for } 0<x \leq 1 \\ I_{\nu}(x) \leq C \mathrm{e}^{x} & \text { for } x \geq 1\end{cases}
$$

Lemma 4.3. For any $u>0, a>0$ and $y>0$, it holds that

$$
\rho_{u}^{a}(y) \leq \frac{2 C u}{a^{2}} \quad \text { and } \quad \tilde{\rho}_{u}^{a}(y) \leq C .
$$

For any fixed $u>0$ and $y>0$, it holds that

$$
\tilde{\rho}_{u}^{a}(y) \underset{a \rightarrow \infty}{\longrightarrow} 1 .
$$

Proof. Using (4.3), we easily have (4.10).

If $2 \sqrt{u y} / a \leq 1$, we have

$$
\rho_{u}^{a}(y) \leq C \frac{2 u}{a^{2}} \quad \text { and } \quad \tilde{\rho}_{u}^{a}(y) \leq C .
$$


If $2 \sqrt{u y} / a>1$, we have

$$
\begin{aligned}
& \rho_{u}^{a}(y) \leq C \mathrm{e}^{-(\sqrt{u}+\sqrt{y})^{2} / a} \frac{\sqrt{u / y}}{a} \leq C \frac{2 u}{a^{2}}, \\
& \tilde{\rho}_{u}^{a}(y) \leq C \mathrm{e}^{-(\sqrt{u}+\sqrt{y})^{2} / a} \leq C .
\end{aligned}
$$

Therefore we obtain (4.9).

Lemma 4.4. Let $f \in \mathcal{L}_{+}^{1}, x \in I$ and $u>0$. For any $a \in I$, set

$$
\begin{aligned}
N_{t}^{a, u} & =a \mathbb{P}_{x}\left[f\left(L_{\eta_{u}^{a}}\right) 1_{\left\{t<\eta_{u}^{a}\right\}} \mid \mathcal{F}_{t}\right], \\
M_{t}^{a, u} & =a \mathbb{P}_{x}\left[f\left(L_{\eta_{u}^{a}}\right) \mid \mathcal{F}_{t}\right] .
\end{aligned}
$$

Then it holds that $N_{t}^{a, u} \rightarrow M_{t}^{s, f}$ and $M_{t}^{a, u} \rightarrow M_{t}^{s, f}$ in probability with respect to $\mathbb{P}_{x}$ as $a \rightarrow \infty$, where $M_{t}^{s, f}$ has been defined in (3.2). $M_{t}^{s, f}$.

Proof. In what follows in this section we sometimes write to simplify $M_{t}$ for

(i) By the strong Markov property and by Lemma 4.2, we have, for $a>X_{t}$,

$$
N_{t}^{a, u}=\left.a \mathbb{P}_{X_{t}}\left[f\left(b+L_{\eta_{u-c}^{a}}\right)\right]\right|_{\substack{b=L_{t} \\ c=L_{t}^{a}}} 1_{\left\{t<\eta_{u}^{a}\right\}}=(\mathrm{I})_{a}+(\mathrm{II})_{a},
$$

where

$$
\begin{aligned}
(\mathrm{I})_{a} & =\left.X_{t}\left\{\mathrm{e}^{-(u-c) / a} f(b)+\int_{0}^{\infty} f(b+y) \rho_{u-c}^{a}(y) \mathrm{d} y\right\}\right|_{\substack{b=L_{t} \\
c=L_{t}^{a}}} 1_{\left\{t<\eta_{u}^{a}\right\}}, \\
(\mathrm{II})_{a} & =\left.\left(1-\frac{X_{t}}{a}\right) \int_{0}^{\infty} f(b+y) \tilde{\rho}_{u-c}^{a}(y) \mathrm{d} y\right|_{\substack{b=L_{t} \\
c=L_{t}^{a}}} 1_{\left\{t<\eta_{u}^{a}\right\}} .
\end{aligned}
$$

Letting $a \rightarrow \infty$, we deduce from Lemma 4.3 that in probability with respect to $\mathbb{P}_{x}$

$$
\begin{aligned}
& \text { (I) })_{a \rightarrow \infty} X_{t} f\left(L_{t}\right), \\
& (\mathrm{II})_{a} \underset{a \rightarrow \infty}{\longrightarrow} \int_{0}^{\infty} f\left(L_{t}+y\right) \mathrm{d} y .
\end{aligned}
$$

We thus obtain $N_{t}^{a, u} \rightarrow M_{t}^{s, f}$ in probability with respect to $\mathbb{P}_{x}$. Set

$$
A_{t}^{a, u}=M_{t}^{a, u}-N_{t}^{a, u}=a f\left(L_{\eta_{u}^{a}}\right) 1_{\left\{\eta_{u}^{a} \leq t\right\}} .
$$

Since $A_{t}^{a, u} \rightarrow 0$, we obtain $M_{t}^{a, u} \rightarrow M_{t}^{s, f}$ in probability with respect to $\mathbb{P}_{x}$.

Theorem 4.5. Let $f \in \mathcal{L}_{+}^{1}, x \in I$ and $u>0$.

(i) For any finite stopping time $T$, there is the $\mathcal{L}^{1}$ convergence

$$
N_{T}^{a, u} \underset{a \rightarrow \infty}{\longrightarrow} M_{T}^{s, f} \text { in } \mathcal{L}^{1}\left(\mathbb{P}_{x}\right)
$$


Consequently, for any bounded adapted process $\left(F_{t}\right)$, it holds that

$$
a \mathbb{P}_{x}\left[F_{T} f\left(L_{\eta_{u}^{a}}\right) ; T<\eta_{u}^{a}\right] \underset{a \rightarrow \infty}{\longrightarrow} \mathbb{P}_{x}\left[F_{T} M_{T}^{s, f}\right]
$$

(ii) Assume furthermore that $\ell^{\prime}(=\ell=\infty)$ is either type-1-natural or type-2-natural. Then, for any bounded stopping time $T$, we have

$$
M_{T}^{a, u} \underset{a \rightarrow \infty}{\longrightarrow} M_{T}^{s, f} \text { in } \mathcal{L}^{1}\left(\mathbb{P}_{x}\right)
$$

and, for any bounded adapted process $\left(F_{t}\right)$, it holds that

$$
a \mathbb{P}_{x}\left[F_{T} f\left(L_{\eta_{u}^{a}}\right)\right] \underset{a \rightarrow \infty}{\longrightarrow} \mathbb{P}_{x}\left[F_{T} M_{T}^{s, f}\right]
$$

Proof. (i) By the proof of Lemma 4.4 and by Lemma 4.3, we obtain, for $a>1$,

$$
\begin{aligned}
N_{t}^{a, u} & \leq X_{t} f\left(L_{t}\right)+\left(\frac{2 C u}{a}+C\right) \int_{0}^{\infty} f\left(L_{t}+y\right) \mathrm{d} y \\
& \leq M_{t}^{s, f}+(2 C u+C) \int_{0}^{\infty} f(y) \mathrm{d} y
\end{aligned}
$$

where the last quantity is integrable with respect to $\mathbb{P}_{x}$. Thus we obtain the desired result by the dominated convergence theorem.

(ii) Observe that since $\left(M_{t}^{s, f}\right)$ is a $\mathbb{P}_{x}$-martingale, we may apply the optional stopping theorem to obtain

$$
\begin{aligned}
\mathbb{P}_{x}\left[A_{T}^{a, u}\right] & =\mathbb{P}_{x}\left[X_{\eta_{u}^{a}} f\left(L_{\eta_{u}^{a}}\right) ; \eta_{u}^{a} \leq T\right] \\
& \leq \mathbb{P}_{x}\left[M_{\eta_{u}^{a}} ; \eta_{u}^{a} \leq T\right] \\
& =\mathbb{P}_{x}\left[M_{T} ; \eta_{u}^{a} \leq T\right] \underset{a \rightarrow \infty}{\longrightarrow} 0 .
\end{aligned}
$$

Since $A_{T}^{a, u} \rightarrow 0, \mathbb{P}_{x}$-a.s., we see that $A_{T}^{a, u} \rightarrow 0$ in $\mathcal{L}^{1}\left(\mathbb{P}_{x}\right)$. Hence we obtain (4.11) and (4.12).

\subsection{Limit as $u$ tends to infinity with $a$ being fixed.}

Suppose $\ell^{\prime}(=\ell=\infty)$ is either entrance, type-1-natural or type-2-natural. We thus have, for any $x, a \in I$,

$$
\mathbb{P}_{x}\left(\eta_{u}^{a}<\infty\right)=1 \quad \text { and } \quad \eta_{u}^{a} \underset{u \rightarrow \infty}{\longrightarrow} \infty \mathbb{P}_{x^{-}} \text {a.s. }
$$

In fact, $\eta_{u}^{a}$ increases to a limit $\eta_{\infty}^{a}$ which must be infinite $\mathbb{P}_{x}$-a.s. by (4.1). For the clock $\left(\tau=\eta_{u}^{a}, u>0\right)$, we only consider the weights $f\left(L_{\eta_{u}^{a}}\right)$ for $f(u)=\mathrm{e}^{-\beta u}$ and $f(u)=1_{\{u=0\}}$.

Lemma 4.6. Let $x, a \in I, \beta>0$ and $t>0$. For $u>0$, set

$$
N_{t}^{u, \beta, a}=\mathrm{e}^{\beta u /(1+\beta a)} \mathbb{P}_{x}\left[\mathrm{e}^{-\beta L_{\eta_{u}^{a}}} 1_{\left\{t<\eta_{u}^{a}\right\}} \mid \mathcal{F}_{t}\right], \quad M_{t}^{u, \beta, a}=\mathrm{e}^{\beta u /(1+\beta a)} \mathbb{P}_{x}\left[\mathrm{e}^{-\beta L_{\eta_{u}^{a}}} \mid \mathcal{F}_{t}\right]
$$

and 


$$
M_{t}^{\beta, a}=\frac{1+\beta\left(X_{t} \wedge a\right)}{1+\beta a} \exp \left(-\beta L_{t}+\frac{\beta}{1+\beta a} L_{t}^{a}\right) .
$$

Then it holds that $N_{t}^{u, \beta, a} \rightarrow M_{t}^{\beta, a}$ and $M_{t}^{u, \beta, a} \rightarrow M_{t}^{\beta, a}, \mathbb{P}_{x}$-a.s. as $u \rightarrow \infty$.

Proof. By the strong Markov property and by Lemmas 4.1 and 4.2, we have, for $u$ large enough to have $\eta_{u}^{a}>t$,

$$
\begin{aligned}
N_{t}^{u, \beta, a} & \left.=\mathrm{e}^{\beta u /(1+\beta a)} \exp \left(-\beta L_{t}\right) \mathbb{P}_{X_{t}}\left[\exp \left(-\beta L_{\eta_{u-c}^{a}}\right)\right)\right]\left.\right|_{c=L_{t}^{a}} \\
& =\left\{1_{\left\{a \leq X_{t}\right\}}+\frac{1+\beta X_{t}}{1+\beta a} 1_{\left\{X_{t}<a\right\}}\right\} \exp \left(-\beta L_{t}+\frac{\beta}{1+\beta a} L_{t}^{a}\right) \\
& =M_{t}^{\beta, a} .
\end{aligned}
$$

Thus we obtain $N_{t}^{u, \beta, a} \rightarrow M_{t}^{\beta, a}, \mathbb{P}_{x^{-}}$a.s. as $u \rightarrow \infty$. Since

$$
A_{t}^{u, \beta, a}:=M_{t}^{u, \beta, a}-N_{t}^{u, \beta, a}=\mathrm{e}^{\beta u /(1+\beta a)} \mathrm{e}^{-\beta L_{\eta_{u}^{a}}} 1_{\left\{\eta_{u}^{a} \leq t\right\}},
$$

we have $A_{t}^{u, \beta, a} \rightarrow 0, \mathbb{P}_{x^{-}}$a.s., and thus we obtain $M_{t}^{u, \beta, a} \rightarrow M_{t}^{\beta, a}, \mathbb{P}_{x}$-a.s.

TheOrem 4.7. Let $x, a \in I$ and $\beta>0$. Then, for any $t>0$, it holds that

$$
N_{t}^{u, \beta, a} \underset{u \rightarrow \infty}{\longrightarrow} M_{t}^{\beta, a} \quad \text { and } \quad M_{t}^{u, \beta, a} \underset{u \rightarrow \infty}{\longrightarrow} M_{t}^{\beta, a} \quad \text { in } \mathcal{L}^{1}\left(\mathbb{P}_{x}\right)
$$

Consequently, for any bounded adapted process $\left(F_{t}\right)$, it holds that

$$
\lim _{u \rightarrow \infty} \mathrm{e}^{\beta u /(1+\beta a)} \mathbb{P}_{x}\left[F_{t} \mathrm{e}^{-\beta L_{\eta_{u}^{a}}^{a}} ; t<\eta_{u}^{a}\right]=\lim _{u \rightarrow \infty} \mathrm{e}^{\beta u /(1+\beta a)} \mathbb{P}_{x}\left[F_{t} \mathrm{e}^{-\beta L_{\eta_{u}^{a}}}\right]=\mathbb{P}_{x}\left[F_{t} M_{t}^{\beta, a}\right] .
$$

It also holds that $\left(M_{t}^{\beta, a}\right)$ is a $\mathbb{P}_{x}$-martingale.

Proof. Let us first prove that $\mathbb{P}_{x}\left[\mathrm{e}^{c L_{t}^{a}}\right]<\infty$ for all $c>0$ and $t>0$. Following the same argument as in the proof of Lemma 2.1, we obtain

$$
\mathbb{P}_{a}\left[\exp \left(c L_{\boldsymbol{e}_{q}}^{a}\right)\right]=\frac{1}{r_{q}(a, a)} \int_{0}^{\infty} \mathrm{e}^{c u} \mathrm{e}^{-u / r_{q}(a, a)} \mathrm{d} u .
$$

Since $r_{q}(a, a) \rightarrow 0$ as $q \rightarrow \infty$, we may take $q>0$ large enough so that $r_{q}(a, a)<1 / c$. This shows that $\mathbb{P}_{a}\left[\exp \left(c L_{\boldsymbol{e}_{q}}^{a}\right)\right]<\infty$. By the monotonicity, we see that $\mathbb{P}_{x}\left[\mathrm{e}^{c L_{t}^{a}}\right]<\infty$ for all $t>0$.

The fact that $L_{t}^{a}$ admits exponential moments implies that $M_{t}^{\beta, a} \in \mathcal{L}^{1}\left(\mathbb{P}_{x}\right)$ for all $t>0$. Thus, by the dominated convergence theorem, we see that $N_{t}^{u, \beta, a} \underset{u \rightarrow \infty}{\longrightarrow} M_{t}^{\beta, a}$ in $\mathcal{L}^{1}\left(\mathbb{P}_{x}\right)$ for all $t>0$.

We second note that, for $q>0$,

$$
\mathbb{P}_{x}\left(\eta_{u}^{a} \leq t\right) \leq \mathrm{e}^{q t} \mathbb{P}_{x}\left[\mathrm{e}^{-q \eta_{u}^{a}}\right] \leq \mathrm{e}^{q t} \mathbb{P}_{a}\left[\mathrm{e}^{-q \eta_{u}^{a}}\right]=\mathrm{e}^{q t} \mathrm{e}^{-u / r_{q}(a, a)} .
$$

We may take $q>0$ large enough so that $r_{q}(a, a)<(1+\beta a) / \beta$. Then we obtain 


$$
\mathbb{P}_{x}\left[A_{t}^{u, \beta, a}\right] \leq \mathrm{e}^{\beta u /(1+\beta a)} \mathbb{P}_{x}\left(\eta_{u}^{a} \leq t\right) \leq \mathrm{e}^{q t} \exp \left\{-\left(\frac{1}{r_{q}(a, a)}-\frac{\beta}{1+\beta a}\right) u\right\} \underset{u \rightarrow \infty}{\longrightarrow} 0 .
$$

Thus we obtain $A_{t}^{u, \beta, a} \underset{u \rightarrow \infty}{\longrightarrow} 0$ in $\mathcal{L}^{1}\left(\mathbb{P}_{x}\right)$ for all $t>0$, which implies $M_{t}^{u, \beta, a} \underset{u \rightarrow \infty}{\longrightarrow} M_{t}^{\beta, a}$ in $\mathcal{L}^{1}\left(\mathbb{P}_{x}\right)$ for all $t>0$.

We conclude this section by looking at the weight $1_{\left\{L_{\eta_{u}=0}\right\}}=1_{\left\{\eta_{u}^{a}<T_{0}\right\}}$.

Theorem 4.8. Let $x, a \in I$. For $u>0$ and $t>0$, set

$$
\begin{aligned}
N_{t}^{u, \infty, a} & =\mathrm{e}^{u / a} \mathbb{P}_{x}\left(t<\eta_{u}^{a}<T_{0} \mid \mathcal{F}_{t}\right) \\
M_{t}^{u, \infty, a} & =\mathrm{e}^{u / a} \mathbb{P}_{x}\left(\eta_{u}^{a}<T_{0} \mid \mathcal{F}_{t}\right) \\
M_{t}^{\infty, a} & =\frac{X_{t} \wedge a}{a} \mathrm{e}^{L_{t}^{a} / a} 1_{\left\{t<T_{0}\right\}} .
\end{aligned}
$$

Then,

$$
N_{t}^{u, \infty, a} \underset{u \rightarrow \infty}{\longrightarrow} M_{t}^{\infty, a} \quad \text { and } \quad M_{t}^{u, \infty, a} \underset{u \rightarrow \infty}{\longrightarrow} M_{t}^{\infty, a} \quad \mathbb{P}_{x} \text {-a.s. and in } \mathcal{L}^{1}\left(\mathbb{P}_{x}\right) .
$$

Consequently, for any bounded adapted process $\left(F_{t}\right)$, it holds that

$$
\lim _{u \rightarrow \infty} \mathrm{e}^{u / a} \mathbb{P}_{x}\left[F_{t} ; t<\eta_{u}^{a}<T_{0}\right]=\lim _{u \rightarrow \infty} \mathrm{e}^{u / a} \mathbb{P}_{x}\left[F_{t} ; \eta_{u}^{a}<T_{0}\right]=\mathbb{P}_{x}\left[F_{t} M_{t}^{\infty, a}\right] .
$$

It also holds that $\left(M_{t}^{\infty, a}\right)$ is a $\mathbb{P}_{x}$-martingale.

Proof. Letting $\beta \rightarrow \infty$, we see, from Lemma 4.6 and Theorem 4.7, that

$$
N_{t}^{u, \infty, a}=M_{t}^{\infty, a} 1_{\left\{t<\eta_{u}^{a}\right\}}
$$

and

$$
A_{t}^{u, \infty, a}:=M_{t}^{u, \infty, a}-N_{t}^{u, \infty, a}=\mathrm{e}^{u / a} 1_{\left\{\eta_{u}^{a}<T_{0}\right\}} 1_{\left\{\eta_{u}^{a} \leq t\right\}} .
$$

The remainder of the proof is the same as that of Theorem 4.7.

\section{Universal $\sigma$-finite measures.}

In this section we shall describe the law of some penalized processes using universal $\sigma$-finite measures. We deal with the transient and recurrent cases separately.

\subsection{The transient case.}

Theorem 5.1. Suppose $\ell<\infty$, i.e., 0 is transient. Let $f \in \mathcal{L}_{+}^{1}$ and $x \in I$. Let $t$ be a constant time and let $F_{t}$ be a bounded $\mathcal{F}_{t}$-measurable functional. Then

$$
\lim _{q \downarrow 0} \mathbb{P}_{x}\left[F_{t} f\left(L_{\boldsymbol{e}_{q}}\right) ; t<\boldsymbol{e}_{q}\right]=\lim _{q \downarrow 0} \mathbb{P}_{x}\left[F_{t} f\left(L_{\boldsymbol{e}_{q}}\right)\right]=\mathbb{P}_{x}\left[F_{t} f\left(L_{\infty}\right)\right] .
$$

If, in particular, $\ell$ is type-3-natural (see Section 7), then 


$$
\lim _{a \uparrow \ell} \mathbb{P}_{x}\left[F_{t} f\left(L_{T_{a}}\right) ; t<T_{a}\right]=\lim _{a \uparrow \ell} \mathbb{P}_{x}\left[F_{t} f\left(L_{T_{a}}\right)\right]=\mathbb{P}_{x}\left[F_{t} f\left(L_{\infty}\right)\right] .
$$

Proof. By Theorem 2.4, we see that (5.1) is equivalent to

$$
\mathbb{P}_{x}\left[F_{t} f\left(L_{\infty}\right)\right]=\mathbb{P}_{x}\left[F_{t} M_{t}\right],
$$

where

$$
M_{t}=\frac{1}{\ell}\left\{X_{t} f\left(L_{t}\right)+\left(1-\frac{X_{t}}{\ell}\right) \int_{0}^{\infty} \mathrm{e}^{-u / \ell} f\left(L_{t}+u\right) \mathrm{d} u\right\} .
$$

On the other hand, we use (i) of Theorem 2.2 and obtain

$$
\mathbb{P}_{x}\left[f\left(L_{\infty}\right) \mid \mathcal{F}_{t}\right]=\left.\mathbb{P}_{X_{t}}\left[f\left(a+L_{\infty}\right)\right]\right|_{a=L_{t}}=M_{t}
$$

Thus we obtain (5.3)

Using Theorem 3.3 instead of Theorem 2.4, we can obtain (5.2) in the same way as above.

REMARK 5.2. Observe that both penalizations yield the same measure $\mathbb{Q}_{x}$

$$
\mathbb{Q}_{x}=\frac{f\left(L_{\infty}\right)}{\mathbb{P}_{x}\left[f\left(L_{\infty}\right)\right]} \cdot \mathbb{P}_{x},
$$

which is absolutely continuous with respect to $\mathbb{P}_{x}$. This is not very surprising since the initial process spends little time at 0 , hence the penalization by the local time at 0 has no real impact on the measure $\mathbb{P}_{x}$.

\subsection{The recurrent case.}

Let $\mathbb{P}_{x, y}^{(u)}$ denote the law of the bridge with duration $u$ starting from $x$ and ending at $y$. Following [6], this measure can be characterized by

$$
\mathbb{P}_{x, y}^{(u)}(A)=\mathbb{P}_{x}\left[1_{A} \frac{p_{u-t}\left(X_{t}, y\right)}{p_{u}(x, y)}\right], \quad A \in \mathcal{F}_{t}, 0<t<u,
$$

where $p_{u}(x, y)$ denotes the transition density of the process $X$ with respect to $\mathrm{d} m(y)$. We have the conditioning formula:

$$
\mathbb{P}_{x}\left[\int_{0}^{\infty} F_{u} \mathrm{~d} L_{u}\right]=\int_{0}^{\infty} \mathbb{P}_{x}\left[\mathrm{~d} L_{u}\right] \mathbb{P}_{x, 0}^{(u)}\left[F_{u}\right]
$$

for all non-negative predictable processes $\left(F_{u}\right)$, where we write symbolically (see ItôMcKean [7, p.183]):

$$
\mathbb{P}_{x}\left[\mathrm{~d} L_{u}\right]=p_{u}(x, 0) \mathrm{d} u .
$$

We also have the last exit decomposition formula (see Biane-Yor [2]):

$$
\mathbb{P}_{x}\left[F_{t} ; T_{0} \leq t\right]=\int_{0}^{t} \mathbb{P}_{x}\left[\mathrm{~d} L_{u}\right]\left(\mathbb{P}_{x, 0}^{(u)} \bullet \boldsymbol{n}^{[t-u]}\right)\left[F_{t}\right]
$$


for all non-negative $\mathcal{F}_{t}$-measurable functionals $\left(F_{t}\right)$, where $\bullet$ denotes the concatenation operator and

$$
\boldsymbol{n}^{[t]}(\cdot)=\boldsymbol{n}\left(\cdot \cap\left\{t<T_{0}\right\}\right)
$$

For $h=h_{0}($ see $(1.6))$ or $h=s$ (the scale function), let $\mathbb{P}_{x}^{h}$ denote the law of the $h$-transform:

$$
\begin{aligned}
& \mathbb{P}_{x}^{h}(A ; t<\zeta)=\frac{1}{h(x)} \mathbb{P}_{x}\left[1_{A \cap\left\{t<T_{0}\right\}} h\left(X_{t}\right)\right] \quad(x>0), \\
& \mathbb{P}_{0}^{h}(A ; t<\zeta)=\boldsymbol{n}\left[1_{A} h\left(X_{t}\right)\right]
\end{aligned}
$$

for $A \in \mathcal{F}_{t}$ and where $\zeta$ denotes the lifetime of the process. Note that, when $h=h_{0}$ or $h=s$, the coordinate process under $\mathbb{P}_{x}^{h}$ never hits zero; see [19, Theorems 7.6 and 7.3]. We now define the $\sigma$-finite measure

$$
\mathcal{P}_{x}^{h}=\int_{0}^{\infty} \mathbb{P}_{x}\left[\mathrm{~d} L_{u}\right]\left(\mathbb{P}_{x, 0}^{(u)} \bullet \mathbb{P}_{0}^{h}\right)+h(x) \mathbb{P}_{x}^{h} .
$$

TheOREM 5.3. Suppose $\ell=\infty$, i.e., 0 is recurrent. Let $f \in \mathcal{L}_{+}^{1}$ and $x \in I$. Let $t$ be a constant time and let $F_{t}$ be a bounded $\mathcal{F}_{t}$-measurable functional. Then

$$
\lim _{q \downarrow 0} H(q) \mathbb{P}_{x}\left[F_{t} f\left(L_{\boldsymbol{e}_{q}}\right) ; t<\boldsymbol{e}_{q}\right]=\mathcal{P}_{x}^{h_{0}}\left[F_{t} f\left(L_{\zeta}\right) ; t<\zeta\right] .
$$

Proof. By Theorem 2.4, it suffices to show

$$
\mathcal{P}_{x}^{h_{0}}\left[F_{t} f\left(L_{\zeta}\right) ; t<\zeta\right]=\mathbb{P}_{x}\left[F_{t} N_{t}^{h_{0}, f}\right] .
$$

Denote $g=\sup \left\{t<\zeta: X_{t}=0\right\}$, where $\sup \emptyset=0$. Observe first that on the set $\{0=g \leq t<\zeta\}$, we have

$$
\begin{aligned}
\mathcal{P}_{x}^{h_{0}}\left[F_{t} f\left(L_{\zeta}\right) ; 0=g \leq t<\zeta\right] & =h_{0}(x) \mathbb{P}_{x}^{h_{0}}\left[F_{t} f\left(L_{t}\right) ; t<\zeta\right] \\
& =\mathbb{P}_{x}\left[F_{t} f\left(L_{t}\right) h_{0}\left(X_{t}\right) ; t<T_{0}\right] .
\end{aligned}
$$

Next, on the set $\{0<g \leq t<\zeta\}$, we have

$$
\begin{aligned}
\mathcal{P}_{x}^{h_{0}}\left[F_{t} f\left(L_{\zeta}\right) ; 0<g \leq t<\zeta\right] & =\int_{0}^{t} \mathbb{P}_{x}\left[\mathrm{~d} L_{u}\right]\left(\mathbb{P}_{x, 0}^{(u)} \bullet \mathbb{P}_{0}^{h}\right)\left[F_{t} f\left(L_{t}\right) ; t<\zeta\right] \\
& =\int_{0}^{t} \mathbb{P}_{x}\left[\mathrm{~d} L_{u}\right]\left(\mathbb{P}_{x, 0}^{(u)} \bullet \boldsymbol{n}\right)\left[F_{t} f\left(L_{t}\right) h_{0}\left(X_{t}\right)\right] \\
& =\mathbb{P}_{x}\left[F_{t} f\left(L_{t}\right) h_{0}\left(X_{t}\right) ; T_{0} \leq t\right] .
\end{aligned}
$$

Finally, on the set $\{t<g<\zeta\}$, we have

$$
\mathcal{P}_{x}^{h_{0}}\left[F_{t} f\left(L_{\zeta}\right) ; t<g<\zeta\right]=\int_{t}^{\infty} \mathbb{P}_{x}\left[\mathrm{~d} L_{u}\right] \mathbb{P}_{x, 0}^{(u)}\left[F_{t} f\left(L_{u}\right)\right]
$$




$$
\begin{aligned}
& =\mathbb{P}_{x}\left[F_{t} \int_{t}^{\infty} f\left(L_{u}\right) \mathrm{d} L_{u}\right] \\
& =\mathbb{P}_{x}\left[F_{t} \int_{L_{t}}^{\infty} f(u) \mathrm{d} u\right] .
\end{aligned}
$$

Summing all three terms yields (5.5).

TheOREM 5.4. Suppose $\ell^{\prime}$ is either entrance, type-1-natural or type-2-natural. Let $f \in \mathcal{L}_{+}^{1}$ and $x \in I$. Let $t$ be a constant time and let $F_{t}$ be a bounded $\mathcal{F}_{t}$-measurable functional. Then

$$
\lim _{a \uparrow \ell} a \mathbb{P}_{x}\left[F_{t} f\left(L_{T_{a}}\right) ; t<T_{a}\right]=\mathcal{P}_{x}^{s}\left[F_{t} f\left(L_{\zeta}\right) ; t<\zeta\right] .
$$

The proof is similar to that of Theorem 5.3, where we use Theorem 3.3 instead of Theorem 2.4, so we omit it.

REMARK 5.5. Define, for $h=h_{0}$ or $h=s$, the penalized measures

$$
\mathbb{Q}_{x}^{h, f}(A ; t<\zeta)=\mathbb{P}_{x}\left[1_{A} \frac{N_{t}^{h, f}}{N_{0}^{h, f}}\right] \quad\left(\text { for } A \in \mathcal{F}_{t}\right) .
$$

Looking at (5.4), we see that, under the assumptions of Theorems 5.3 or 5.4, the paths of the coordinates processes under $\mathbb{Q}_{x}^{h, f}$ are essentially given, up to some killing time $\zeta$, by the concatenation of a weighted bridge of the original diffusion, and a process conditioned not to hit 0 . In particular, the penalized process is no longer recurrent, even if $\zeta=\infty$, i.e. if $N^{h, f}$ is a $\mathbb{P}_{x}$-martingale. Of course, in this case, the two probability measures $\mathbb{Q}_{x}^{h, f}$ and $\mathbb{P}_{x}$ are singular.

\section{Exponential weights.}

Let us investigate the example where we take

$$
f(x)=\mathrm{e}^{-c x}, \quad c>0 .
$$

In this specific case, the penalized process remains a generalized diffusion, which is not the case with other functions $f$. The supermartingales $N_{t}=N_{t}^{h_{0}, f}$ and $N_{t}=M_{t}^{s, f}$, which have been given by (2.5) and (3.2), respectively, may be represented at the same time as

$$
N_{t}=h^{c}\left(X_{t}\right) \mathrm{e}^{-c L_{t}}
$$

where $h=h_{0}$ and $s$, respectively, and

$$
h^{c}(x)=h(x)+\frac{1-(x / \ell)}{c+(1 / \ell)} .
$$

Since $\left(N_{t}\right)$ is a supermartingale, we may define the subprobability measure $\mathbb{Q}_{x}^{h, c}$ by 


$$
\mathbb{Q}_{x}^{h, c}(A ; t<\zeta)=\mathbb{P}_{x}\left[\frac{h^{c}\left(X_{t}\right)}{h^{c}(x)} \mathrm{e}^{-c L_{t}} ; A\right] \quad \text { for } A \in \mathcal{F}_{t} \text { and } t \geq 0 .
$$

Then the process $\left\{X,\left(\mathbb{Q}_{x}^{h, c}\right)_{x \in I}\right\}$ is a diffusion on $I$ whose local generator on $\left(0, \ell^{\prime}\right)$ without killing part is given as $\left(h^{c}\right)^{-1}(\mathrm{~d} / \mathrm{d} m)(\mathrm{d} / \mathrm{d} s) h^{c}$. Thus the corresponding speed measure and scale function are given as

$$
m^{h, c}(x)=\int_{(0, x]} h^{c}(y)^{2} \mathrm{~d} m(y), \quad s^{h, c}(x)=\int_{0}^{x} \frac{\mathrm{d} y}{h^{c}(y)^{2}} .
$$

Denote $\rho_{q}=\phi_{q}-\psi_{q} / H(q)$ and

$$
\phi_{q}^{h, c}=h^{c}(0) \cdot \frac{\phi_{q}+c \psi_{q}}{h^{c}}, \quad \rho_{q}^{h, c}=h^{c}(0) \cdot \frac{\rho_{q}}{h^{c}} .
$$

Then we obtain (see Theorems 7.3 and 7.6 of $[\mathbf{1 9}]$ ) that $\varphi=\phi_{q}^{h, c}\left(\right.$ resp. $\rho_{q}^{h, c}$ ) is a positive increasing (resp. decreasing) solution to the differential equation

$$
\left(\frac{\mathrm{d}}{\mathrm{d} m^{h, c}} \frac{\mathrm{d}}{\mathrm{d} s^{h, c}}-\frac{\pi_{0}}{h^{c}}\right) \varphi=q \varphi\left(\text { if } h=h_{0}\right), \quad\left(\frac{\mathrm{d}}{\mathrm{d} m^{h, c}} \frac{\mathrm{d}}{\mathrm{d} s^{h, c}}\right) \varphi=q \varphi(\text { if } h=s)
$$

which satisfies the boundary condition

$$
\phi_{q}^{h, c}(0)=1 \quad \text { and } \quad \frac{\mathrm{d} \phi_{q}^{h, c}}{\mathrm{~d} s^{h, c}}(0)=0 \quad\left(\text { resp. } \rho_{q}^{h, c}(0)=1\right) .
$$

Note that we have used here the values

$$
h^{c}(0)=\frac{1}{c+(1 / \ell)} \quad \text { and } \quad\left(h^{c}\right)^{\prime}(0)=\frac{c}{c+(1 / \ell)} .
$$

THEOREM 6.1. The resolvent operator for the diffusion $\left\{X,\left(\mathbb{Q}_{x}^{h, c}\right)_{x \in I}\right\}$ is given as

$$
\mathbb{Q}_{x}^{h, c}\left[\int_{0}^{\infty} \mathrm{e}^{-q t} f\left(X_{t}\right) \mathrm{d} t\right]=\int_{I} r_{q}^{h, c}(x, y) f(y) \mathrm{d} m^{h, c}(y), \quad q>0,
$$

where

$$
r_{q}^{h, c}(x, y)=r_{q}^{h, c}(y, x)=\frac{H(q)}{h^{c}(0)^{2}(c H(q)+1)} \phi_{q}^{h, c}(x) \rho_{q}^{h, c}(y), \quad x, y \in I, x \leq y .
$$

Consequently, 0 for $\left\{X,\left(\mathbb{Q}_{x}^{h, c}\right)_{x \in I}\right\}$ is regular-reflecting.

Proof. Let $\varphi^{c}(x)=\varphi(x) h^{c}(x)$. Then we have

$$
\begin{aligned}
\mathbb{P}_{x} & {\left[\int_{0}^{\infty} \mathrm{e}^{-q t} \varphi^{c}\left(X_{t}\right) \mathrm{e}^{-c L_{t}} \mathrm{~d} t\right] } \\
& =\mathbb{P}_{x}\left[\int_{0}^{T_{0}} \mathrm{e}^{-q t} \varphi^{c}\left(X_{t}\right) \mathrm{d} t\right]+\mathbb{P}_{x}\left[\mathrm{e}^{-q T_{0}}\right] \mathbb{P}_{0}\left[\int_{0}^{\infty} \mathrm{e}^{-q t} \varphi^{c}\left(X_{t}\right) \mathrm{e}^{-c L_{t}} \mathrm{~d} t\right]
\end{aligned}
$$




$$
\begin{aligned}
& =R_{q}^{0} \varphi^{c}(x)+\mathbb{P}_{x}\left[\mathrm{e}^{-q T_{0}}\right] \mathbb{P}_{0}\left[\sum_{u} \mathrm{e}^{-c u-q \eta_{u-}^{0}} \int_{0}^{T_{0}(p(u))} \mathrm{e}^{-q t} \varphi^{c}\left(p(u)_{t}\right) \mathrm{d} t\right] \\
& =R_{q}^{0} \varphi^{c}(x)+\mathbb{P}_{x}\left[\mathrm{e}^{-q T_{0}}\right] \mathbb{P}_{0}\left[\int_{0}^{\infty} \mathrm{e}^{-c u-q \eta_{u}^{0}} \mathrm{~d} u\right] \boldsymbol{n}\left[\int_{0}^{T_{0}} \mathrm{e}^{-q t} \varphi^{c}\left(X_{t}\right) \mathrm{d} t\right] \\
& =R_{q}^{0} \varphi^{c}(x)+\mathbb{P}_{x}\left[\mathrm{e}^{-q T_{0}}\right] \cdot \frac{1}{c+\frac{1}{H(q)}} \cdot \frac{R_{q} \varphi^{c}(0)}{H(q)} .
\end{aligned}
$$

Since $\mathbb{P}_{x}\left[\mathrm{e}^{-q T_{0}}\right] R_{q} \varphi^{c}(0)=R_{q} \varphi^{c}(x)-R_{q}^{0} \varphi^{c}(x)$, we obtain

$$
\mathbb{Q}_{x}^{h, c}\left[\int_{0}^{\infty} \mathrm{e}^{-q t} \varphi\left(X_{t}\right) \mathrm{d} t\right]=\frac{1}{h^{c}(x)}\left\{\frac{1}{c H(q)+1} R_{q} \varphi^{c}(x)+\frac{c H(q)}{c H(q)+1} R_{q}^{0} \varphi^{c}(x)\right\} .
$$

From this we obtain (6.1).

REMARK 6.2. The boundary classification at $\ell^{\prime}$ is the same as that for the $h$ transform of the stopped process; see Theorems 7.3 and 7.6 of [19].

\section{Appendix: the boundary classification.}

\begin{tabular}{|c|c|c|}
\hline & $\ell=\infty$ & $\ell<\infty$ \\
\hline $\begin{array}{l}m(\infty)=\infty \\
\pi_{0}=0\end{array}$ & $\begin{array}{l}\text { (1) } 0 \text { is null-recurrent } \\
{\left[\ell^{\prime}=\ell=\infty\right]} \\
\ell^{\prime} \text { is type-1-natural }\end{array}$ & $\begin{array}{l}\text { (3) } 0 \text { is transient } \\
{\left[\ell^{\prime}<\ell<\infty\right]} \\
\ell^{\prime} \text { is regular-elastic } \\
{\left[\ell^{\prime}=\ell<\infty\right]} \\
\ell^{\prime} \text { is regular-absorbing } \\
\quad \text { exit } \\
\quad \text { type-3-natural }\end{array}$ \\
\hline $\begin{array}{l}m(\infty)<\infty \\
\pi_{0}>0\end{array}$ & $\begin{array}{l}\text { (2) } 0 \text { is positive recurrent } \\
{\left[\ell^{\prime}<\ell=\infty\right]} \\
\ell^{\prime} \text { is regular-reflecting } \\
{\left[\ell^{\prime}=\ell=\infty\right]} \\
\ell^{\prime} \text { is entrance } \\
\quad \text { type-2-natural }\end{array}$ & [impossible] \\
\hline
\end{tabular}

The following tables explain the boundary classification which we take from [19] and the recurrence property of the corresponding diffusion to each class:

\begin{tabular}{lllll}
$x=\ell^{\prime}$ & & $I^{\prime}$ & $I$ & $x=0$ \\
\hline regular-reflecting & $\ell^{\prime}<\ell=\infty$ & {$\left[0, \ell^{\prime}\right]$} & $=I^{\prime}$ & positive recurrent \\
regular-elastic & $\ell^{\prime}<\ell<\infty$ & {$\left[0, \ell^{\prime}\right]$} & {$\left[0, \ell^{\prime}\right] \cup\{\ell\}$} & transient \\
regular-absorbing & $\ell^{\prime}=\ell<\infty$ & {$[0, \ell)$} & {$[0, \ell]$} & transient \\
exit & $\ell^{\prime}=\ell<\infty$ & {$[0, \ell)$} & {$[0, \ell]$} & transient \\
entrance & $\ell^{\prime}=\ell=\infty$ & {$[0, \infty)$} & $=I^{\prime}$ & positive recurrent \\
type-1-natural & $\ell^{\prime}=\ell=\infty$ & {$[0, \infty)$} & $=I^{\prime}$ & null recurrent \\
type-2-natural & $\ell^{\prime}=\ell=\infty$ & {$[0, \infty)$} & $=I^{\prime}$ & positive recurrent \\
type-3-natural & $\ell^{\prime}=\ell<\infty$ & {$[0, \ell)$} & $=I^{\prime}$ & transient
\end{tabular}


As the reader may not be familiar with our classification of boundaries, it may be useful to give below some examples of computation of boundaries. Let $\tilde{X}$ be a diffusion on $[0, \infty)$ where 0 is the reflecting boundary and whose local generator on $(0, \infty)$ is given by

$$
\tilde{L} f=\frac{1}{2}\left(f^{\prime \prime}-b f^{\prime}\right)=\frac{\mathrm{d}}{\mathrm{d} \tilde{m}} \frac{\mathrm{d}}{\mathrm{d} \tilde{s}} f \quad \text { on } C_{c}((0, \infty))
$$

for some function $b$ of the form $b(x)=c x^{\nu-1}$, which we may call the power drift. Then its scale change $X=\tilde{s}(\tilde{X})$ is a diffusion with natural scale $s(x)=x$ and with speed measure $\mathrm{d} m(x)$ defined by $m=\tilde{m} \circ \tilde{s}^{-1}$.

(i) Let $\alpha$ be a constant and

$$
\tilde{L} f=\frac{1}{2} f^{\prime \prime}-\frac{2 \alpha-1}{2 x} f^{\prime}=\frac{\mathrm{d}}{\mathrm{d} \tilde{m}} \frac{\mathrm{d}}{\mathrm{d} \tilde{s}} f \quad \text { on } C_{c}((0, \infty)),
$$

where we may choose $\tilde{m}(x)=2 /(2-2 \alpha) x^{2-2 \alpha}$ and $\tilde{s}(x)=1 /(2 \alpha) x^{2 \alpha}$. The corresponding diffusion is called the reflecting Bessel process of index $\alpha$. As we require that 0 is regular-reflecting, we assume $0<\alpha<1$. If we take $m=\tilde{m} \circ \tilde{s}^{-1}$, then it falls into the case (1) above.

(ii) Let $c$ and $\nu$ be non-zero constants and

$$
\tilde{L} f=\frac{1}{2}\left(f^{\prime \prime}-c \nu x^{\nu-1} f^{\prime}\right) \quad \text { on } C_{c}((0, \infty)) .
$$

If $\nu=1$, then it is a Brownian motion with constant negative drift. If $\nu=2$, then it is an Ornstein-Uhlenbeck process. As we require that 0 is regular-reflecting, we assume $c>0$ and $\nu>0$. In this case we may choose

$$
s^{\prime}=\mathrm{e}^{c x^{\nu}}, \quad s=\int_{0}^{x} \mathrm{e}^{c y^{\nu}} \mathrm{d} y
$$

and

$$
m^{\prime}=2 \mathrm{e}^{-c x^{\nu}}, \quad m=2 \int_{0}^{x} \mathrm{e}^{-c y^{\nu}} \mathrm{d} y .
$$

In particular, we have $m(\infty)<\infty$. Note that

$$
\begin{aligned}
J:=\frac{1}{2} \int_{1}^{\infty}\{s(x)-s(1)\} \mathrm{d} m(x) & =\int_{1}^{\infty}\left(\int_{1}^{x} \mathrm{e}^{c y^{\nu}} \mathrm{d} y\right) \mathrm{e}^{-c x^{\nu}} \mathrm{d} x \\
& =\int_{1}^{\infty}\left(\int_{y}^{\infty} \mathrm{e}^{-c x^{\nu}} \mathrm{d} x\right) \mathrm{e}^{c y^{\nu}} \mathrm{d} y
\end{aligned}
$$

We shall prove that

$$
\infty \text { is }\left\{\begin{array}{l}
\text { type-2-natural if } 0<\nu \leq 2, \\
\text { entrance if } 2<\nu<\infty
\end{array}\right.
$$

which is equivalent as saying that $J=+\infty$ (resp. $J<+\infty)$, see $[\mathbf{1 9}]$. 
If $1 \leq \nu \leq 2$, then

$$
\begin{aligned}
\int_{1}^{x} \mathrm{e}^{c y^{\nu}} \mathrm{d} y & =\int_{1}^{x}\left(\mathrm{e}^{c y^{\nu}}\right)^{\prime} \frac{y^{1-\nu}}{c \nu} \mathrm{d} y \\
& =\left[\mathrm{e}^{c y^{\nu}} \frac{y^{1-\nu}}{c \nu}\right]_{1}^{x}+\frac{\nu-1}{c \nu} \int_{1}^{x} \mathrm{e}^{c y^{\nu}} y^{-\nu} \mathrm{d} y \\
& \geq \mathrm{e}^{c x^{\nu}} \frac{x^{1-\nu}}{c \nu}-c^{\prime}
\end{aligned}
$$

for some constant $c^{\prime}>0$. Hence we have

$$
J \geq \frac{1}{c \nu} \int_{1}^{\infty} x^{1-\nu} \mathrm{d} x-c^{\prime} \int_{1}^{\infty} \mathrm{e}^{-c x^{\nu}} \mathrm{d} x=\infty .
$$

For $\nu>0$, we have

$$
\begin{aligned}
\int_{y}^{\infty} \mathrm{e}^{-c x^{\nu}} \mathrm{d} x & =-\int_{y}^{\infty}\left(\mathrm{e}^{-c x^{\nu}}\right)^{\prime} \frac{x^{1-\nu}}{c \nu} \mathrm{d} y \\
& =-\left[\mathrm{e}^{-c x^{\nu}} \frac{x^{1-\nu}}{c \nu}\right]_{y}^{\infty}+\frac{1-\nu}{c \nu} \int_{y}^{\infty} \mathrm{e}^{-c x^{\nu}} x^{-\nu} \mathrm{d} x .
\end{aligned}
$$

If $0<\nu<1$, then

$$
\int_{y}^{\infty} \mathrm{e}^{-c x^{\nu}} \mathrm{d} x \geq \mathrm{e}^{-c y^{\nu}} \frac{y^{1-\nu}}{c \nu} \quad \text { and } \quad J \geq \frac{1}{c \nu} \int_{1}^{\infty} y^{1-\nu} \mathrm{d} y=\infty .
$$

If $\nu>2$, then

$$
\int_{y}^{\infty} \mathrm{e}^{-c x^{\nu}} \mathrm{d} x \leq \mathrm{e}^{-c y^{\nu}} \frac{y^{1-\nu}}{c \nu} \quad \text { and } \quad J \leq \frac{1}{c \nu} \int_{1}^{\infty} y^{1-\nu} \mathrm{d} y<\infty .
$$

\section{References}

[1] J. Azéma and M. Yor, Une solution simple au problème de Skorokhod (French), In: Séminaire de Probabilités, XIII, Lecture Notes in Math., 721, Springer, Berlin, 1979, 90-115.

[2] P. Biane and M. Yor, Valeurs principales associées aux temps locaux browniens (French), Bull. Sci. Math. (2), 111 (1987), 23-101.

[3] R. M. Blumenthal, Excursions of Markov processes, Probab. Appl., Birkhäuser Boston Inc., Boston, MA, 1992 .

[4] L. Chaumont and R. A. Doney, On Lévy processes conditioned to stay positive, Electron. J. Probab., 10 (2005), 948-961.

[5] P. Debs, Penalisation of the standard random walk by a function of the one-sided maximum, of the local time, or of the duration of the excursions, In: Séminaire de probabilités XLII, Lecture Notes in Math., 1979, Springer, Berlin, 2009, 331-363.

[6] P. Fitzsimmons, J. Pitman and M. Yor, Markovian bridges: construction, Palm interpretation, and splicing, In: Seminar on Stochastic Processes, 1992 (Seattle, WA, 1992), Progr. Probab., 33, Birkhäuser Boston, Boston, MA, 1993, 101-134.

[ 7 ] K. Itô and H. P. McKean, Diffusion processes and their sample paths, Springer-Verlag, Berlin-New York, 1974

[ 8 ] F. B. Knight, Brownian local times and taboo processes, Trans. Amer. Math. Soc., 143 (1969), 
$173-185$.

[ 9 ] N. N. Lebedev, Special functions and their applications, Dover Publications, Inc., New York, 1972. Revised edition, translated from the Russian and edited by Richard A. Silverman, Unabridged and corrected republication.

[10] J. Najnudel, B. Roynette and M. Yor, A global view of Brownian penalisations, MSJ Memoirs, 19, Mathematical Society of Japan, Tokyo, 2009.

[11] C. Profeta, Penalizing null recurrent diffusions, Electron. J. Probab., 17 (2012), no. 69, 23 pp.

[12] C. Profeta, Penalization of a positively recurrent diffusion by an exponential function of its local time, Publ. Res. Inst. Math. Sci., 46 (2010), 681-718.

[13] D. Revuz and M. Yor, Continuous martingales and Brownian motion, Springer-Verlag, Berlin, 1999.

[14] B. Roynette, P. Vallois and M. Yor, Limiting laws associated with Brownian motion perturbed by its maximum, minimum and local time, II, Studia Sci. Math. Hungar., 43 (2006), 295-360.

[15] B. Roynette, P. Vallois and M. Yor, Limiting laws associated with Brownian motion perturbed by normalized exponential weights, I, Studia Sci. Math. Hungar., 43 (2006), 171-246.

[16] B. Roynette and M. Yor, Penalising Brownian paths, Lecture Notes in Math., 1969, SpringerVerlag, Berlin, 2009.

[17] P. Salminen and P. Vallois, On subexponentiality of the Lévy measure of the diffusion inverse local time; with applications to penalizations, Electron. J. Probab., 14 (2009), no. 67, 1963-1991.

[18] S. Watanabe, On time inversion of one-dimensional diffusion processes, Z. Wahrscheinlichkeitstheorie und Verw. Gebiete, 31 (1974/75), 115-124.

[19] K. Yano and Y. Yano, On $h$-transforms of one-dimensional diffusions stopped upon hitting zero, In: In memoriam Marc Yor-Séminaire de Probabilités XLVII, Lecture Notes in Math., 2137, Springer, Cham, 2015, 127-156.

[20] K. Yano, Y. Yano and M. Yor, Penalising symmetric stable Lévy paths, J. Math. Soc. Japan, 61 (2009), 757-798.

\section{Christophe Profeta}

Université d'Evry Val-d'Essonne

E-mail: christophe.profeta@univ-evry.fr

\section{Kouji Yano}

Graduate School of Science

Kyoto University

E-mail: kyano@math.kyoto-u.ac.jp

Yuko YANO

Faculty of Science

Kyoto Sangyo University

E-mail: yyano@cc.kyoto-su.ac.jp 